\title{
Vulnerability studies in the fields of transportation and complex networks: a citation network analysis
}

\author{
Kashin Sugishita ${ }^{1,2}$ (D) Yasuo Asakura ${ }^{2}$
}

Accepted: 6 August 2020 / Published online: 29 September 2020

(c) The Author(s) 2020

\begin{abstract}
In recent years, studies on network vulnerability have grown rapidly in the fields of transportation and complex networks. Even though these two fields are closely related, their overall structure is still unclear. In this study, to add clarity comprehensively and objectively, we analyze a citation network consisting of vulnerability studies in these two fields. We collect publication records from an online publication database, the Web of Science, and construct a citation network where nodes and edges represent publications and citation relations, respectively. We analyze the giant weakly connected component consisting of 705 nodes and 4,584 edges. First, we uncover main research domains by detecting communities in the network. Second, we identify major research development over time in the detected communities by applying main path analysis. Third, we quantitatively reveal asymmetric citation patterns between the two fields, which implies that mutual understanding between them is still lacking. Since these two fields deal with the vulnerability of network systems in common, more active interdisciplinary studies should have a great potential to advance both fields in the future.
\end{abstract}

Keywords Network vulnerability · Transportation · Complex networks · Citation network analysis

\section{Introduction}

Our society is surrounded by a great variety of network systems such as power grids, communication systems, water supply systems, gas pipelines, or transportation systems. The quality of our lives relies on the service level of these critical

Kashin Sugishita

kashinsu@buffalo.edu

1 University at Buffalo, Mathematics Building, Room 313, Buffalo, NY 14260-2900, USA

2 Tokyo Institute of Technology, Midorigaoka Building \#1, Room 206A, M1-20, 2-12-1,

O-okayama, Meguro-ku, Tokyo 152-8552, Japan 
infrastructures. As Rinaldi et al. (2001) describe, critical infrastructures are mutually dependent, if they have implemented an even higher level of their services.

However, recent natural and man-made disasters such as floods, hurricanes, tornadoes, volcanic eruptions, earthquakes, tsunamis, or terrorist attacks have revealed an inherent weakness of these interdependent systems. According to Helbing (2013), as the complexity and interaction strength increase, these systems can become unstable, creating uncontrollable situations. In other words, failures can spread out in multiple systems because of the dependency among different systems.

A transportation system is one of the critical infrastructures. In the transportation field, in the 1990s and the beginning of the 2000s, researchers mainly focused on transport network reliability (e.g., Bell and Iida 1997; Asakura 1999; Bell 2000; Chen et al. 2002; Nicholson et al. 2003). Typically, studies on the transport network reliability consider both, the occurrence probability and the consequences. However, according to Taylor (2017), around the beginning of the 2000s, researchers started to discuss something different from reliability: transport network vulnerability. Studies on the transport network vulnerability focus on catastrophic events that rarely occur but bring about devastating consequences on the society. These studies consider only the consequence without the occurrence probability, because the expected values cannot capture the characteristics of such catastrophic events (e.g., D'Este and Taylor 2003; Taylor and D'Este 2007; Taylor 2008). In this way, transport network vulnerability emerged as an important area in the transportation field.

At almost the same time as the emergence of the transport network vulnerability, a new scientific field called complex networks (also known as network science) appeared after two pioneering works (Watts and Strogatz 1998; Barabási and Albert 1999). This field especially focuses on topological characteristics of various realworld network systems. In complex networks, vulnerability is also an important topic. So far, a large number of studies have investigated network vulnerability, especially about influences of the removals of nodes/edges on the performance of the network systems.

As already pointed out in a review paper by Mattsson and Jenelius (2015), one root in the transport network vulnerability is complex networks. In other words, the vulnerability of transport networks has been studied from the perspectives of the network topology. It seems that concepts and methods in these two fields are closely related and interdisciplinary studies between them should have a great potential to advance both fields. However, an overall structure is currently lacking. Before discussing the possibility of more active interdisciplinary studies, it is necessary to grasp how these two fields have evolved over time and how they constitute the current structure. In the present study, to understand the overall structure of the vulnerability studies in these two fields, we analyze a citation network where nodes and edges represent publications and their citation relations, respectively. This approach enables us to obtain the entire picture of the vulnerability studies in a comprehensive and objective manner. Our focus is to understand the entire structure of the previous studies rather than scientometric indexes of individual papers, researchers, and journals (e.g., Heilig and Voß 2015; Jiang et al. 2020; Modak et al. 2019).

The rest of this paper is organized as follows. We propose a framework for analyzing a citation network in Sect. 2. In Sect. 3, we apply the proposed framework 
to our problem which aims to understand the overall structure of the vulnerability studies and discuss the obtained results. This paper ends with conclusions in Sect. 4.

\section{Methods}

In this section, we propose a framework for analyzing citation networks. The proposed framework can be applied to any topic in any research field. It consists of six steps as shown in Fig. 1.

\subsection{Data collection}

As the first step, we collect publication records from an online publication database, the Web of Science Core Collection (hereinafter referred to as WoS). We perform a search in the WoS with "network" and "vulnerability" as keywords. Next, we refine publications into the five WoS categories: 1) transportation science technology, 2) transportation, 3) engineering multidisciplinary, 4) physics multidisciplinary, and 5) multidisciplinary sciences. Then we further refine publications into four types of documents in the WoS: 1) article, 2) proceedings paper, 3) book chapter, and 4) review.

\subsection{Construction of a citation network}

As the second step, we construct a citation network where nodes and directed edges represent publications and citation relations, respectively. We construct a citation network not only from the publications obtained from the WoS described

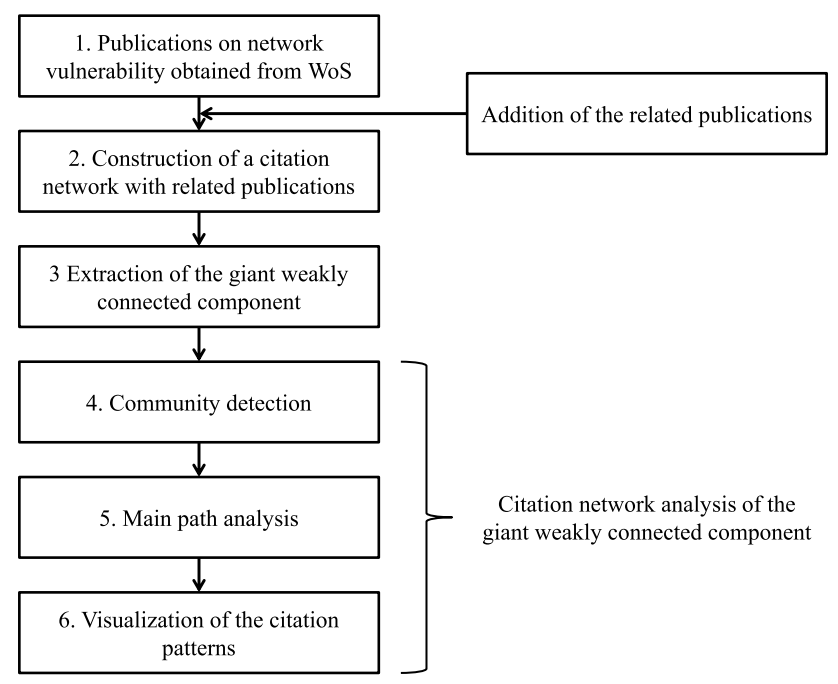

Fig. 1 The proposed framework for analyzing a citation network 
in the previous section, but also from related publications. The related publications are defined as the ones which are cited by more than 10 publications in the data set obtained from the WoS. We use CitNetExplorer (Van Eck and Waltman 2014) to add these related publications. The reason why we add the related publications is to consider publications which are not directly related to network vulnerability, but present important concepts for the vulnerability studies (e.g., books that present fundamental concepts for the transport network analysis).

\subsection{Extraction of the giant weakly connected component}

As the third step, we extract the giant weakly connected component (hereafter referred to as GWCC) from the constructed network. A weakly connected component is a component where at least one path exists between any pair of nodes without considering the direction of edges. The GWCC is the largest component among all weakly connected components in a network.

\subsection{Community detection}

As the fourth step, we perform a community detection algorithm to the extracted GWCC. In general, a community in a network is a dense part in the network. Therefore, community detection enables us to objectively identify main research domains in a citation network. In this study, we use an algorithm called Louvain algorithm (see Blondel et al. 2008).

The Louvain algorithm aims to maximize a quality function for community detection, modularity $Q$, which is defined as follows:

$$
Q=\frac{1}{2 m} \sum_{i j}\left[A_{i j}-\frac{k_{i} k_{j}}{2 m}\right] \delta\left(C_{i}, C_{j}\right)
$$

where $A_{i j}$ is the weight of the edge between nodes $i$ and $j, k_{i}\left(=\sum_{j} A_{i j}\right)$ is the sum of the weights of the edges attached to node $i, C_{i}$ is the community that node $i$ belongs to, $\delta\left(C_{i}, C_{j}\right)$ is 1 if $C_{i}=C_{j}$ (nodes $i$ and $j$ belong to the same community) and 0 otherwise, and $m=\left(\sum_{i j} A_{i j}\right) / 2$. It is noted that for an unweighted graph, the weight of an edge is one and the total weights of a node equals to its degree (the number of edges attached to the node). Modularity $Q$ takes a value between -1 and 1 . Networks with high modularity have dense connections between the nodes within communities but sparse connections between nodes in different communities. In other words, a higher value of modularity $Q$ implies a better quality of community detection.

The Louvain algorithm is an agglomerative algorithm which works by taking a node and joining it into groups, and joining groups with other groups and so on. This algorithm consists of two steps and they are repeated iteratively (Barabási 2016). 


\subsubsection{Step 1}

The algorithm starts with a weighted network with $N$ nodes, initially assigning each node to a different community. For each node $i$, we evaluate the increase of the modularity if we place node $i$ in the community of one of its adjacent nodes $j$. Then we move node $i$ into the community where the modularity increases most (and it is also a positive value). If all of the increased modurality is negative, node $i$ stays in its original community. We repeat this process until no more improvement of the modularity is achieved.

The change of the modularity $\Delta Q$ which is obtained by a node $i$ into a community $C$ is calculated as follows:

$$
\begin{aligned}
\Delta Q & =\left[\frac{\sum_{i n}+2 k_{i, i n}}{2 W}-\left(\frac{\sum_{t o t}+k_{i}}{2 W}\right)^{2}\right]-\left[\frac{\sum_{i n}}{2 W}-\left(\frac{\sum_{t o t}}{2 W}\right)^{2}-\left(\frac{k_{i}}{2 W}\right)^{2}\right] \\
& =\frac{k_{i, i n}}{W}-\frac{k_{i} \sum_{t o t}}{2 W^{2}}
\end{aligned}
$$

where $\sum_{i n}$ is the sum of the weights of edges inside of the community $C, \sum_{\text {tot }}$ is the sum of the weights of edges of all nodes in the community $C, k_{i}$ is the sum of the weights of the edges of node $i, k_{i, i n}$ is the sum of the weights of the edges of node $i$ to nodes in the community $C$, and $W$ is the sum of the weights of all edges in the whole network.

\subsubsection{Step 2}

A new network can be constructed based on the identified communities in step 1. The communities obtained in step 1 are aggregated. In other words, nodes belonging to the same community are merged into one node. This generates selfloops which correspond to edges between nodes in the same community. Once step 2 is completed, steps 1 and 2 are repeated until no further improvement of the modularity is achieved.

\subsection{Main path analysis}

As the fifth step, we perform the so-called main path analysis (Hummon and Dereian 1989) to each of the detected communities. The main path analysis is used to uncover the time-series major paths in a citation network.

A publication always cites already existing publications. As shown in Fig. 2, we define sources and sinks as publications with zero in-degree (not cited by other publications) and zero out-degree (not citing other publications), respectively. 


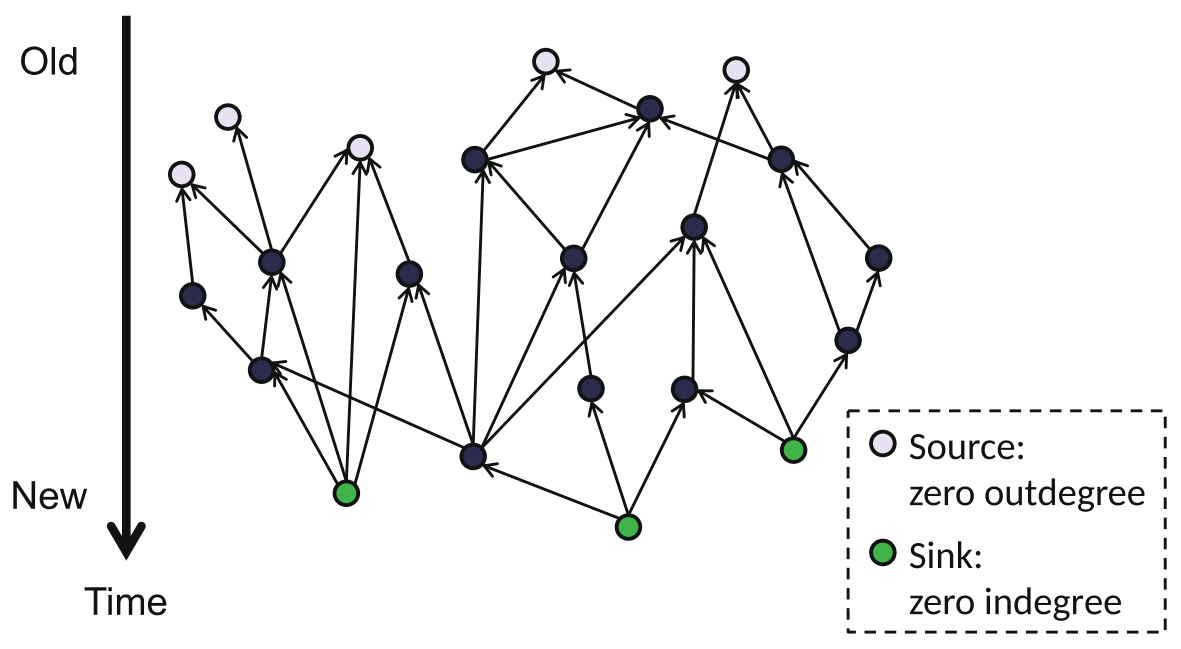

Fig. 2 An illustrative example of a citation network

\subsubsection{Step 1}

As the first step of the main path analysis, we calculate the search path count for each edge (Batagelj 2003). The search path count is the number of times the edge is traversed through all possible combinations between sources and sinks. The logic behind using this index is that if an edge occupies a route through which more knowledge flows, it should have a certain importance in the knowledge-dissemination process (Liu and Lu 2012).

\subsubsection{Step 2}

As the second step, main paths are identified based on the traversal counts of each edge. Specifically, we apply global key-route search (Liu and Lu 2012). The global key-route search is conducted as follows: (1) to specify edges with the certain fraction of the highest traversal counts, (2) to search forward from the specified edges toward sources to identify a path that has the largest overall traversal counts, (3) to search backward from the specified edges toward sinks to identify a path that has the largest overall traversal counts, and (4) to connect paths from sinks to sources including the specified edges. By definition, multiple main paths can be identified. In a simple example of a citation network shown in Fig. 3, we can identify the edge with the highest search path count (the thickest edge) and identify the two main paths from this edge.

\subsection{Citation patterns among different communities}

As the sixth step, we visualize the number of intra/inter-community edges by using a Sankey diagram. Sankey diagrams are a type of flow diagram in which the width of the arrows is proportional to the flow rate (Schmidt 2008). An intra-community edge 


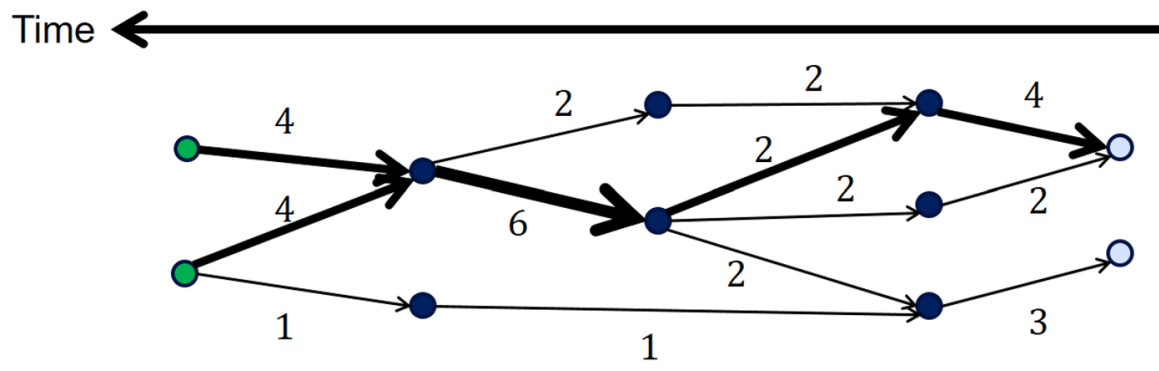

Fig. 3 Main paths in a simple example of a citation network. The number for each edge represents the search path count. The chains of the thick edges represent the main paths

is an edge connecting two nodes in the same community and an inter-community edge is an edge connecting two nodes in different communities. This visualization makes it possible to understand knowledge flow among different communities.

\section{Results and discussions}

Now the proposed framework is applied to our problem, which aims to understand the overall structure of the vulnerability studies in the fields of transportation and complex networks.

\subsection{Descriptive statistics of publication records}

In this section, we show some descriptive statistics of the publications obtained from the WoS. We collect 1,034 publication records which have "network" and "vulnerability" as keywords. This data collection was performed on August 18th, 2018.

\subsubsection{Distribution of publication year}

In Fig. 4, we show the distribution of the published items by year from 1993 to 2018 (25 years span). This figure indicates that the number of studies on the network vulnerability has grown rapidly after 2000 .

\subsubsection{Distribution of document types}

In the WoS, each publication can be classified into one or more types of documents. The obtained data set involves four types of documents including article, proceedings paper, book chapter, and review. Most records are classified into article and/or proceedings paper (Table 1). 


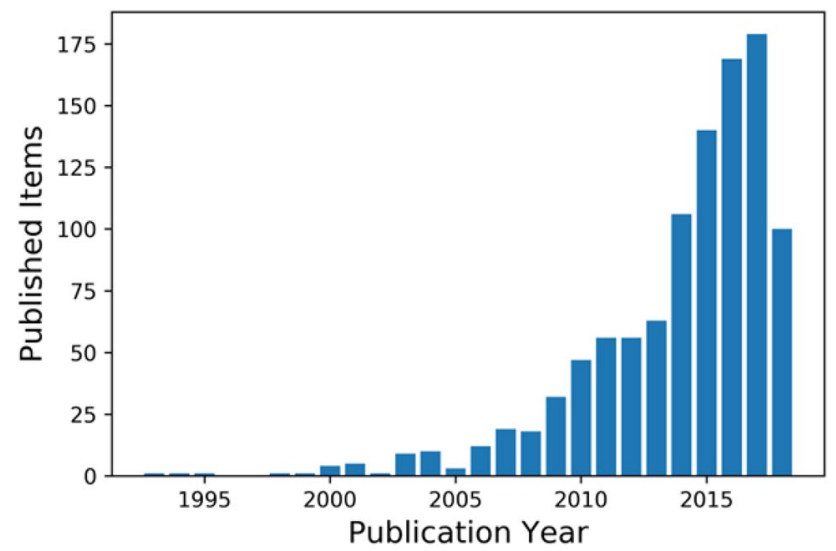

Fig. 4 The distribution of publication year

Table 1 The distribution of document types

\begin{tabular}{lc}
\hline Document type & Publications \\
\hline Article & 779 \\
Proceedings paper & 257 \\
Book chapter & 18 \\
Review & 16 \\
\hline
\end{tabular}

Table 2 The distribution of journals

\begin{tabular}{lc}
\hline Journal title & Publications \\
\hline PLoS ONE & 137 \\
Physica A: Statistical Mechanics and Its Applications & 76 \\
Scientific Reports & 42 \\
Transportation Research Record & 32 \\
Proceedings of the National Academy of Sciences of the USA & 26 \\
Transportation Research Part A: Policy and Practice & 25 \\
Computer-Aided Civil and Infrastructure Engineering & 18 \\
International Journal of Critical Infrastructure Protection & 18 \\
Networks and Spatial Economics & 17 \\
Journal of Transport Geography & 16 \\
\hline
\end{tabular}

\subsubsection{Distribution of journals}

In Table 2, we show the distribution of articles in the top 10 journals. 407 articles $(52.2 \%)$ are published in these 10 journals. Some journals cover a broad area of scientific fields such as PLoS ONE (17.6\%), Scientific Reports (5.39\%), and Proceedings of the National Academy of Sciences of the USA (3.34\%). 76 papers (9.76\%) 
are published in Physica A: Statistical Mechanics and Its Applications, which covers complex systems or network theory. Several journals in the transportation field are listed such as Transportation Research Record (4.11\%), Transportation Research Part A: Policy and Practice (3.21\%), and Journal of Transport Geography (2.05\%). Networks and Spatial Economics (2.18\%) also covers topics about transportation networks. Computer-Aided Civil and Infrastructure Engineering (2.31\%) is intended to act as a bridge between advances in computer technology and civil and infrastructure engineering. International Journal of Critical Infrastructure Protection (2.31\%) covers all areas of critical infrastructure protection, which is a concept relating to the preparedness or response to serious incidents on infrastructure systems.

\subsubsection{Distribution of countries}

In Table 3, we show the top 15 countries about the authors' affiliations. The number of publications are counted by authors' affiliations of each paper. For example, when an article is written by two authors whose affiliations are in the United States and China, it is counted for both countries.

\subsection{Constructed network}

\subsubsection{Visualization}

We construct a citation network with 1181 nodes (1034 publications from the WoS and 147 related publications) and 4601 directed edges. In Fig. 5, we visualize the constructed network. This network has many disconnected components. Specifically, it has 462 weakly connected components and the largest one (GWCC) has 705 nodes and 4584 edges.

Table 3 The distribution of countries

\begin{tabular}{lc}
\hline County & Publications \\
\hline United States & 312 \\
China & 256 \\
Italy & 85 \\
United Kingdom & 78 \\
Australia & 55 \\
South Korea & 48 \\
Netherlands & 46 \\
India & 42 \\
Spain & 40 \\
France & 34 \\
Germany & 32 \\
Sweden & 29 \\
Canada & 28 \\
Japan & 28 \\
Switzerland & 27 \\
\hline
\end{tabular}




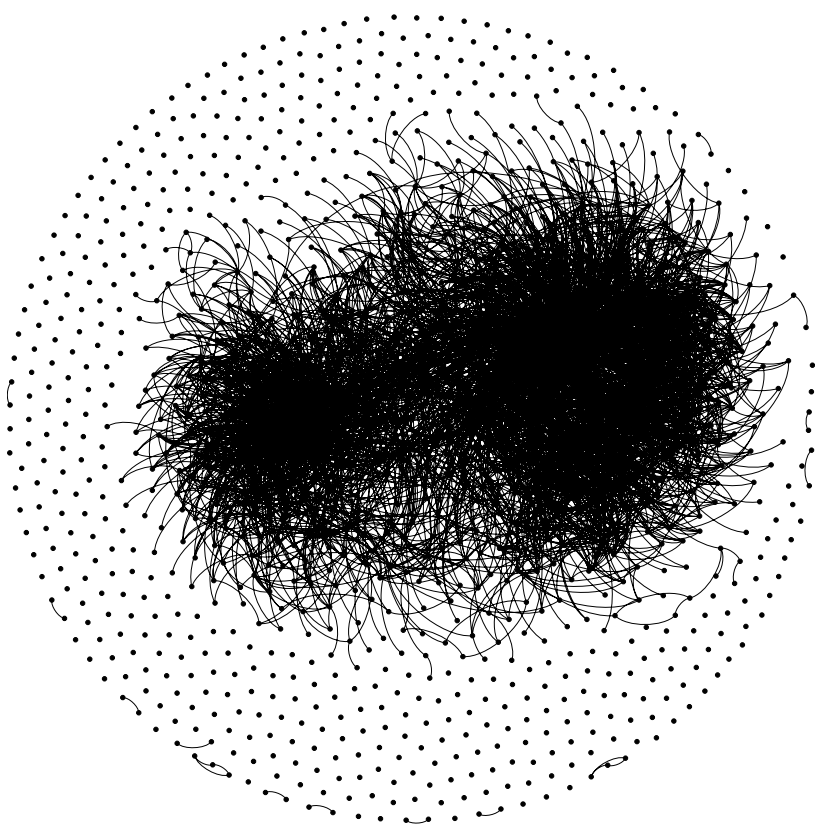

Fig. 5 The constructed network

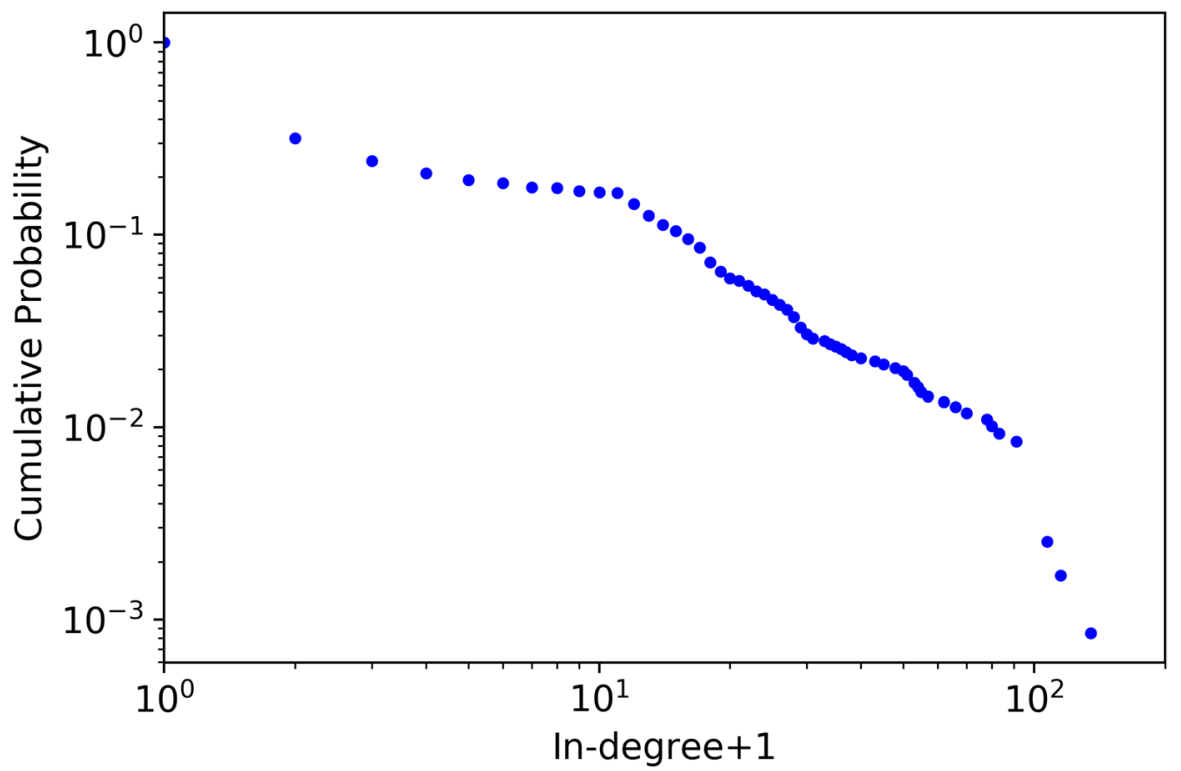

Fig. 6 The cumulative in-degree distribution in the log-log scale 


\subsubsection{Cumulative in-degree distributions}

Here we show the cumulative in-degree distribution on the log-log plot in Fig. 6. In-degree of a node is the number of edges directed into that node. In a citation network, in-degree represents the number of citations of the publication. From Fig. 6, we can observe a straight line in the log-log plot. This indicates that the in-degree distribution follows an approximate power-law distribution. In other words, most papers have a few citations, but a few papers have many citations (the most cited paper by Albert et al. (2000) has 134 citations in this data set).

\subsection{Giant weakly connected component}

We extract and analyze the GWCC in the constructed network. The GWCC has 705 nodes and 4584 directed edges. This means that $59.7 \%$ (705 out of 1181) of all nodes and $99.6 \%(4,584$ out of 4,601) of all edges are included in the GWCC.

\subsection{Community structure in the giant weakly connected component}

We identify seven communities: (1) transport vulnerability, (2) interdependent networks, (3) topological vulnerability, (4) cascading failures, (5) metro and maritime networks, (6) resilience, and (7) scattered community. When two communities locate closely in Fig. 7, this means that the publications in these two communities tend to cite each other. The size of each node represents the in-degree of the node. The color of each node represents the community the node belongs to. The value of

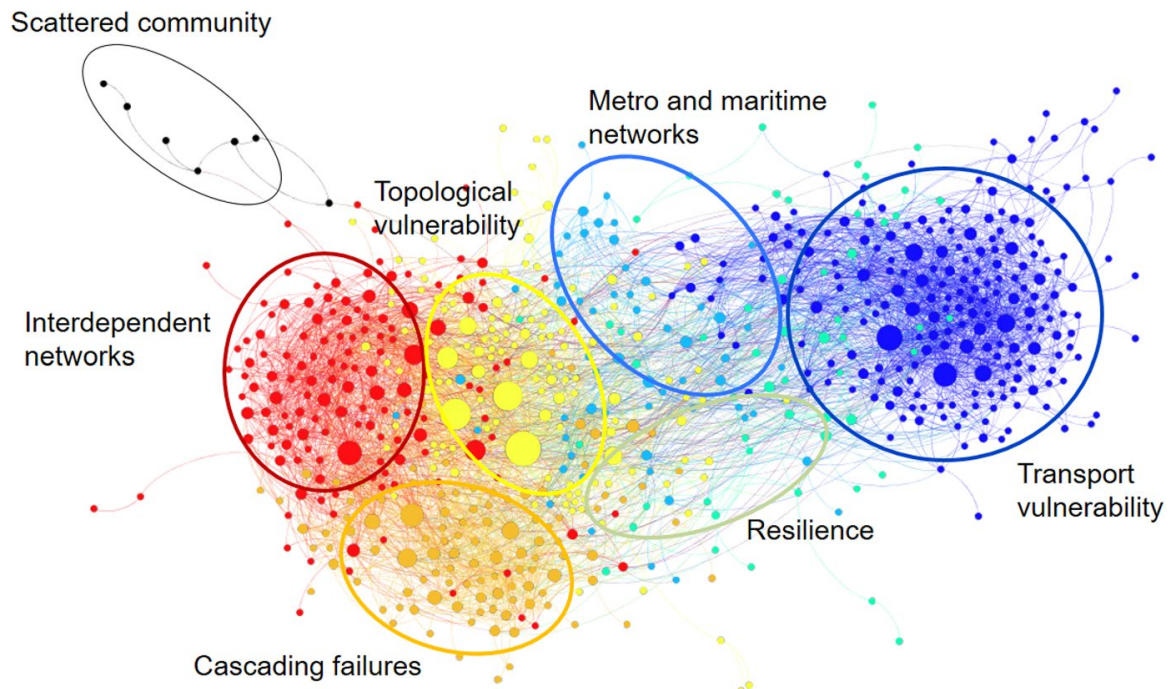

Fig. 7 The community structure in the giant weakly connected component 
Table 4 The number of nodes and intra-community edges in each community

\begin{tabular}{llcc}
\hline Community & Color & Publications & $\begin{array}{l}\text { Intra- } \\
\text { community } \\
\text { edges }\end{array}$ \\
\hline Transport vulnerability & & 208 & 1225 \\
Interdependent networks & Blue & 141 & 738 \\
Topological vulnerability & Red & 127 & 364 \\
Cascading failures & Yellow & 110 & 525 \\
Metro and maritime networks & Orange & 58 & 133 \\
Resilience & Light blue & 54 & 101 \\
Scattered community & Light green & 7 & 7 \\
& Black & 705 & 3093 \\
\hline
\end{tabular}

the modularity $Q$ is 0.461 . According to Fortunato and Barthelemy (2007), this is large enough to consider that the network has community structures.

We show the number of nodes and intra-community edges in each community in Table 4. The community size (the number of nodes) ranges from 7 to 208 and the number of intra-community edges connecting two nodes in the same community ranges from 7 to 1225 . The sum of the number of nodes in communities is 705 , because each node belongs to only one community in the Louvain algorithm. The total number of the intra-community edges is 3093, which indicates $67.5 \%$ of all edges in the GWCC are the intra-community edges.

\subsection{Main paths in each community}

We conduct the main path analysis for all the communities except for the scattered community. In this study, the edges with the top $10 \%$ highest search path count are specified at the first step in the main path analysis. For example, if a citation network has 200 edges, 20 edges are specified and main paths are identified from these edges. In the following sections, we show the obtained main paths in each community and briefly describe how research has been developing there over time.

\subsubsection{Transport vulnerability}

We show the main paths in the transport vulnerability community in Fig. 8. The identified main paths consist of 26 out of 208 publications.

There are three sources (nodes with zero out-degree) in the main paths (Bell and Iida 1997; Chen et al. 1999; D'Este and Taylor 2001). During the 1990s, the transport network reliability became an important research topic (Taylor 2017). Bell and Iida (1997) present fundamental concepts about transport network analysis including the transport network reliability. Chen et al. (1999) propose a concept of capacity reliability which is defined as the probability where the network capacity can accommodate a certain demand at a required service level. Standard approaches on the transport network reliability consider both the occurrence probability and the 


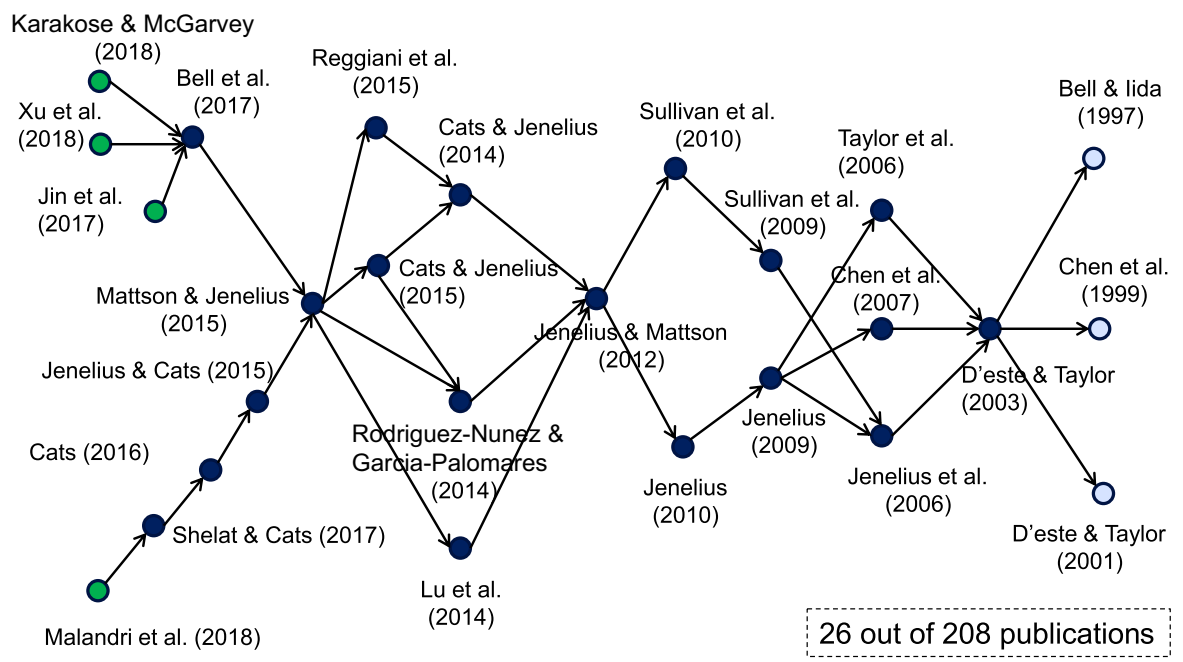

Fig. 8 Papers on transport vulnerability main paths

consequences. On the other hand, D'Este and Taylor (2001) show that such measures do not work well in some situations where the occurrence probability is very small (but not zero) but the consequence is catastrophic with an example of the Australian National Highway System. This indicates the necessity of a new concept of the transport network vulnerability, which is different from the transport network reliability.

After the realization of the necessity of the concept of the transport network vulnerability, D'Este and Taylor (2003) define the vulnerability for nodes and links: (1) a node is vulnerable if a small number of links significantly decreases the accessibility of the node, and (2) a link is critical if the loss of the link significantly decreases the accessibility of the network or of particular nodes. Then the identification of critical parts in transport networks is of great interest. Taylor et al. (2006) discuss three different measures to evaluate the decrease of accessibility using the Australian National Transport Network road system as an example. Jenelius et al. (2006) introduce link importance indices and site exposure indices based on the increase in generalized cost under closures of links. Chen et al. (2007) present network-based accessibility measures considering both of the demand and supply sides.

There are two streams toward a study by Jenelius and Mattsson (2012). One stream starts from a paper by Jenelius (2009). Different from the above-mentioned studies, Jenelius (2009) focuses on the vulnerability not at the link level but at the regional level. Jenelius (2009) adopts two different views on the vulnerability: (1) a region is important if the consequences of a disruption in this region are severe for the whole network (a view from the supply perspective), and (2) a region is exposed if the consequences of a disruption somewhere in the network are severe for the users in the region (a view from a demand perspective). These regional importance and exposure measures are extended from ones proposed by Jenelius et al. (2006). 
Jenelius (2010) discusses the importance of links as backup alternatives in the degraded transportation networks with the concepts of redundancy importance.

The other stream starts from a paper by Sullivan et al. (2009). They review studies on the vulnerability analysis of transportation networks. They show that the performance metrics, terminology, methods in the vulnerability studies can vary dramatically depending on the application or research goals. Sullivan et al. (2010) then propose a method to identify the most critical links and evaluate the network robustness with an index which can be used to compare networks with different sizes, topology, and connectivity levels.

The two streams are integrated by Jenelius and Mattsson (2012). They present a method to analyze the vulnerability of transportation networks under area-covering disruptions such as flooding, snowfall, or storms. Lu et al. (2014) analyze the vulnerability of road networks in the case of flooding. They study inter-city travels and how people adopt to flooding impacts in Bangladesh.

Papers described above mainly focus on the vulnerability of road networks. On the other hand, from the middle of the 2010s, the vulnerability of public transport networks (PTNs) has been investigated intensively. Cats and Jenelius (2014) develop a method to evaluate the dynamic vulnerability for PTNs. They extend a concept of centrality measure in complex networks, edge betweenness centrality, and propose two formulations considering dynamic and stochastic natures of PTNs. This is a successful example of an integration of concepts in transportation fields and complex networks. Rodríguez-N., E. and García-P., J. C. (2014) also investigate the vulnerability of PTNs, using the subway network in Madrid as an example. They show that circular lines play an important role for the network robustness. In addition, a strategy of capacity enhancement to mitigate impacts of unexpected disruptions in PTNs is proposed by Cats and Jenelius (2015).

There are two review papers in the year of 2015. Reggiani et al. (2015) review 33 papers about vulnerability and resilience in transportation networks. Mattsson and Jenelius (2015) also review studies on vulnerability and resilience. They show that there are two distinct traditions in transport vulnerability studies: (1) topological vulnerability studies which analyze topological characteristics and (2) system-based vulnerability studies which attempt to analyze supply and demand.

From the paper by Mattsson and Jenelius (2015), two streams are observed. One stream is about the vulnerability of road networks. Bell et al. (2017) present capacity weighted spectral partitioning to find potential bottlenecks in large transportation networks. The topics of the three sinks (nodes with zero in-degree) which cite the paper by Bell et al. (2017) include an optimization framework to evaluate vulnerability under disruptions of multiple links ( $\mathrm{Xu}$ et al. 2017), a mixed-integer linear programming to evaluate disruptions of links (Karakose and McGarvey 2018), and the vulnerability of road networks regularly threatened by heavy snow (Jin et al. 2017).

Another stream is about the vulnerability of PTNs. It includes four papers (Jenelius and Cats 2015; Cats 2016; Shelat and Cats 2017; Malandri et al. 2018). These studies aim to assess the vulnerability of PTNs in consideration of the specific natures of PTNs in both of the demand and supply sides. Jenelius and Cats (2015) propose a method to evaluate the value of adding new links for the robustness and 
redundancy of PTNs. Cats (2016) presents a method to evaluate the robustness of alternative network designs. Shelat and Cats (2017) introduce a method of a stochastic user equilibrium for PTNs considering boarding denials at stations and perceived increase in travel time because of on-board crowding. Malandri et al. (2018) present a model of temporal and spatial spill-over effects in PTNs.

Summing up the above, the concept of the transport network vulnerability emerged about the year of 2000 to study catastrophic events that rarely occur but cause catastrophic consequences. The obtained main paths suggest that, in the 2000s, the vulnerability of road networks was mainly studied. These papers especially focus on the identification of critical links and exposed nodes. Then, after the paper by Cats and Jenelius (2014) which extends the concept of edge betweenness centrality in complex networks, the vulnerability of PTNs has been studied intensively. After the review paper by Mattsson and Jenelius (2015), two streams about the vulnerability of road networks and PTNs are observed. The papers in the main paths are mainly published in transportation-related journals, such as Transportation Research Part A: Policy and Practice, Transportation Research Part B: Methodological, and Networks and Spatial Economics.

\subsubsection{Metro and maritime networks}

In Fig. 9, we show the main paths in the community of metro and maritime networks. We identify two disconnected main paths. The upper one represents the main paths on the vulnerability of metro and railway networks. The lower one represents the main paths on the vulnerability of maritime shipping and global energy supply networks.

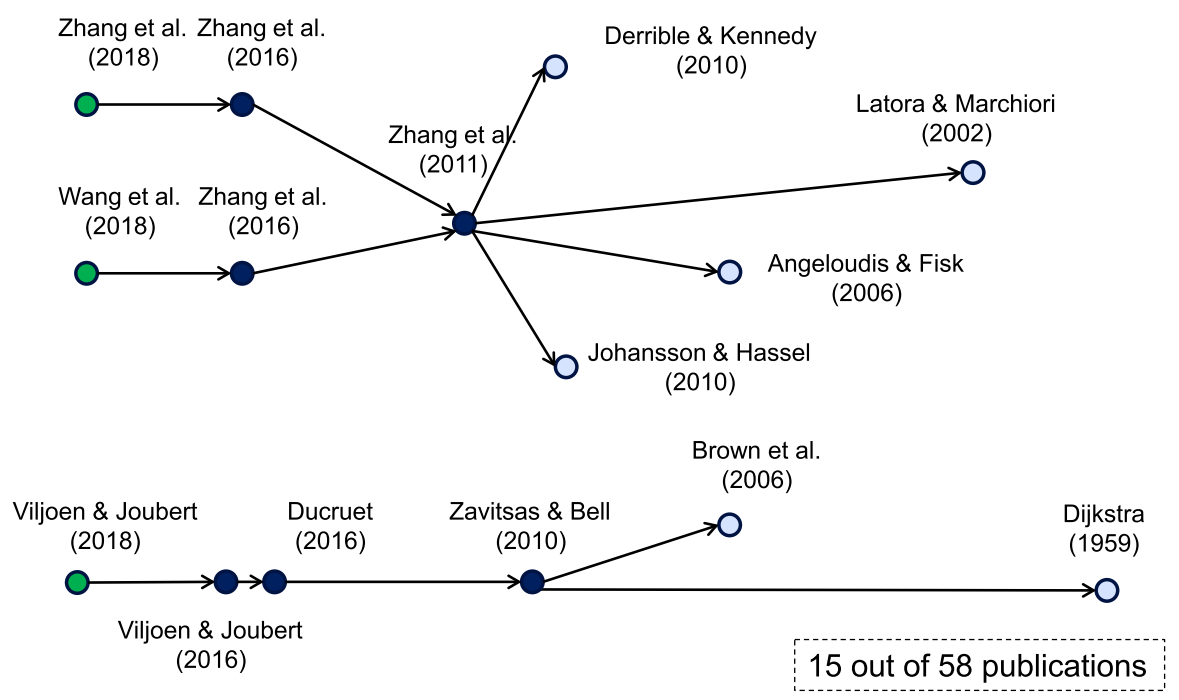

Fig. 9 Papers on metro and maritime networks main paths 
There are four sources in the upper main paths (Latora and Marchiori 2002; Angeloudis and Fisk 2006; Derrible and Kennedy 2010; Johansson and Hassel 2010). All of these studies analyze the vulnerability of subway or railway networks in the perspective of network topology by directly applying the methods in complex networks. Latora and Marchiori (2002) investigate the topological properties of the MBTA (Boston underground transportation system) and bus network. They show that MBTA+ bus network is a small-world network with high efficiency in both of the global and local scales. Angeloudis and Fisk (2006) analyze the world's largest subway networks. Their results show that subway systems can be classified into two classes of networks with exponential degree distributions which are robust to random failures. Derrible and Kennedy (2010) also analyze 33 metro networks in the world and show these networks are scale-free and small-world networks. Johansson and Hassel (2010) investigate the vulnerability of a railway network consisting of five systems from the perspective of interdependent networks.

Zhang et al. (2011) study the vulnerability of Shanghai subway network in China. They show that the subway network is robust to random failures but fragile to targeted attacks, especially to the highest betweenness node-based attacks. There are two streams from this paper. One stream starts from a paper by Zhang et al. (2016b). They conduct a vulnerability analysis on the high speed railway networks in China, the US, and Japan. They show that high speed railway networks are very fragile to malicious attacks. Xu et al. (2018) investigate the evolutionary process of China's high-speed rail network by applying complex networks. The other stream begins with Zhang et al. (2016a). They study the urban rail transit networks by analyzing the hub structures in the networks in consideration with transfers. Zhang et al. (2018) then investigate the topological vulnerability of three metro networks in Guangzhou, Beijing, and Shanghai.

In the lower main paths, there are two sources (Dijkstra 1959; Brown et al. 2006). Dijkstra (1959) proposes an algorithm to find the shortest paths in a network. Brown et al. (2006) propose models for robust critical infrastructures against terrorist attacks. In this way, these two papers are not directly related to maritime networks, but they present widely-applied concepts. Zavitsas and Bell (2010) propose a method to identify vulnerable components in the global energy supply networks and investigate the optimal network structure. Ducruet (2016) then analyzes the role of two canals, Suez and Panama, from the perspective of network topology. They investigate the effect of removals of the canal-dependent flows from the network by using the method in complex networks. Viljoen and Joubert (2016) also apply the theory in complex networks to analyze the vulnerability of the global container shipping network against targeted attacks on edges. They show that removing the highest betweenness edges would greatly impact the transshipment. They point out that these edges are not always the edges that carry the most traffic and could be overlooked as unimportant. Viljoen and Joubert (2018) investigate the vulnerability of the supply chain networks by applying the concept of multilayered networks in complex networks.

Summarizing the above, we identify the two disconnected main paths: (1) the vulnerability of subway or railway networks and (2) the vulnerability of maritime shipping and global supply chain networks. Interestingly, there is a clear difference 
between papers in the transport vulnerability and metro and maritime networks communities. That is, all of the papers except for two source papers (Dijkstra 1959; Brown et al. 2006) in the metro and maritime networks community directly apply the methods in complex networks and analyze the topological vulnerability. Especially, the topological vulnerability of subway and railway networks have been investigated more than the ones of the other transportation modes. One possible reason is that collecting and handling the data of network topology of subway and railway networks is easier than other transportation modes. On the other hand, the main paths also suggest that studies on maritime networks from the perspectives of network topology are currently limited and further studies are desired in the future. It is noted that, differently from the transport vulnerability community, most papers in the main paths in this community are published in a complex network-related journal, especially 9 out of 15 papers in the main paths are published in Physica A: Statistical Mechanics and Its Applications.

\subsubsection{Resilience}

We show the main paths in the resilience community in Fig. 10. The main paths consist of 16 out of 54 papers.

In the obtained main paths, there are five sources (Holling 1973; Pimm 1984; Bruneau et al. 2003; Murray et al. 2008; Cox et al. 2011). According to Reggiani (2013), there are two different ways to define resilience. One is proposed by Holling (1973) and another is proposed by Pimm (1984). Both of the definitions were originally proposed for ecological systems. The resilience based on definition by Holling (1973) is the perturbation which can be absorbed before the system changes into another state. On the other hand, in the definition by Pimm (1984), the resilience

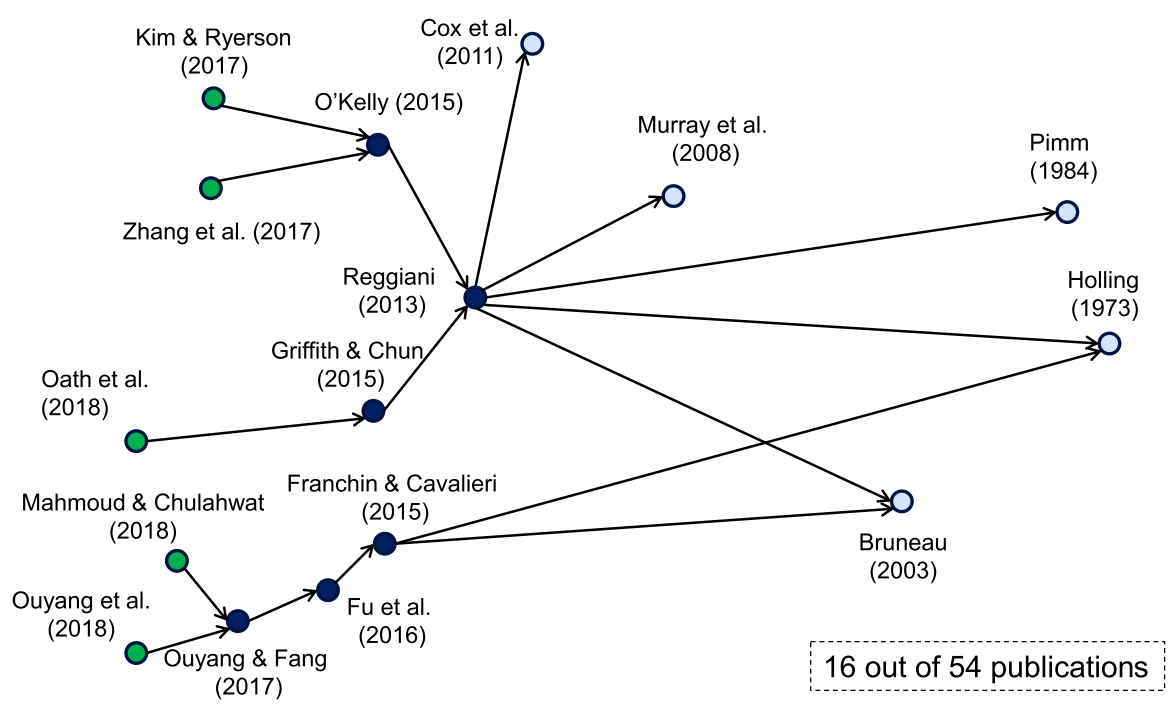

Fig. 10 Papers on resilience main paths 
is measured as the speed of return to equilibrium state. After these two works, in the field of earthquake engineering, Bruneau et al. (2003) present a framework for defining seismic resilience and specifying quantitative measures of resilience. This framework includes three measures of resilience: (1) reduced failure probabilities, (2) reduced consequences from failures, and (3) reduced time to recovery. Murray et al. (2008) present a review of methodologies of vulnerability analysis for infrastructure networks. They explore how different methods impact the infrastructure planning and policy development. Cox et al. (2011) present operational metrics to evaluate resilience of transportation systems. They apply the proposed methods to evaluate the impacts of the London subway bombings in 2007. As described in this paragraph, the source papers include a wide range of fields such as ecological fields, earthquake engineering, and transportation engineering.

Reggiani (2013) proposes a conceptual or methodological framework for analyzing resilience of transportation systems. The author points out that the conditions of hubs can largely affect the entire transportation networks based on studies about the vulnerability of scale-free networks in complex networks. There are two streams from the paper by Reggiani (2013). One stream starts from O'Kelly (2015). This paper studies the resilience of networks with hubs (especially cyber and air passenger/freight networks). Networks whose degree distribution follows power-law have resilience to random attacks, but at the same time they have fragility to targeted attacks. The author concludes that developing mitigation strategies for networks with hub-spoke structures is a critical issue. This paper is cited by two papers (Kim et al. 2017; Zhang et al. 2017). Kim et al. (2017) propose a q-Ad-hoc hub location problem for multi-modal freight transportation systems. This strategy uses alternative hubs in an ad-hoc manner, which give alternative routes satisfying supply and demand in disrupted network systems. Zhang et al. (2017) investigate a hub location problem for resilient power projection networks in military actions. Another stream starts from Griffith and Chun (2015). They study the resilience and vulnerability of economic networks associated with spatial autocorrelation. The sink paper of this stream by Östh et al. (2018) presents a framework for analyzing the mechanism which drives spatial systems from the perspective of the resilience of urban areas.

We can also observe a stream from a paper by Franchin and Cavalieri (2015). They study the seismic resilience using complex network theory. They present an index of resilience based on the evolution of efficiency of communication between citizens during the relocation of the population after an earthquake. Fu et al. (2016) then provide a model which simulates the evolution of infrastructure networks. This model can be used to construct an infrastructure system by reducing cost, increasing efficiency, and improving resilience. Ouyang and Fang (2017) present a framework to optimize resilience of infrastructure systems against intentional attacks. The sink papers of this stream are related to community resilience in consideration of infrastructure, social, and economical aspects (Mahmoud and Chulahwat 2018) and vulnerability to multiple spatially localized attacks on critical infrastructures (Ouyang et al. 2018).

Summing up the above, the knowledge flow about the concept of resilience is visualized in the main paths. Interestingly, the obtained main paths visualize how the concept of resilience has been extended throughout different research fields. That is, 
the resilience was initially conceptualized for ecological systems in the 1970s and 1980s, and they have been developed in other fields including earthquake engineering and transportation engineering. In the same way as the transport vulnerability and metro and maritime networks communities, concept applications from complex networks were observed. Especially the resilience of networks with hubs (e.g., passenger/freight networks (O'Kelly 2015)) has been analyzed based on studies about scale-free networks in complex networks. The main paths seem to be divided into two streams. One stream is related to the resilience of transportation and urban systems, which is shown in the upper part of Fig. 10. These papers are mainly published in Networks and Spatial Economics and Transport Policy. Another stream is about the resilience of critical infrastructure systems against disasters (especially for earthquakes), which is shown in the lower part in Fig. 10. Many papers in this stream are published in Computer-Aided Civil and Infrastructure Engineering.

\subsubsection{Topological vulnerability}

We show the main paths in the topological vulnerability community. The main paths consist of 13 out of 127 publications (Fig. 11).

There are four sources in the obtained main paths (Watts and Strogatz 1998; Barabási and Albert 1999; Barabási et al. 1999; Albert et al. 1999). Two papers, Watts and Strogatz (1998) and Barabási and Albert (1999), are well-known studies. Watts and Strogatz (1998) propose a simple model for generating so-called small-world networks. These networks are not completely regular nor random, but they lie somewhere in between. They found that small-world networks can be highly clustered, but have small average shortest path length. Barabási and Albert (1999) show that many networks in the real world have a common property that their degree distribution follows a power-law. These networks are so-called scale-free networks. They found two generic mechanisms of this type of networks: (1) growth: a network expands by adding new nodes and (2) preferential attachment: new nodes attach preferentially to existing nodes with a high degree. The other two sources, Albert et al. (1999) and Barabási et al. (1999), are also well-cited papers. Albert et al. (1999) analyze the network topology of the World-Wide Web whose nodes and directed edges are

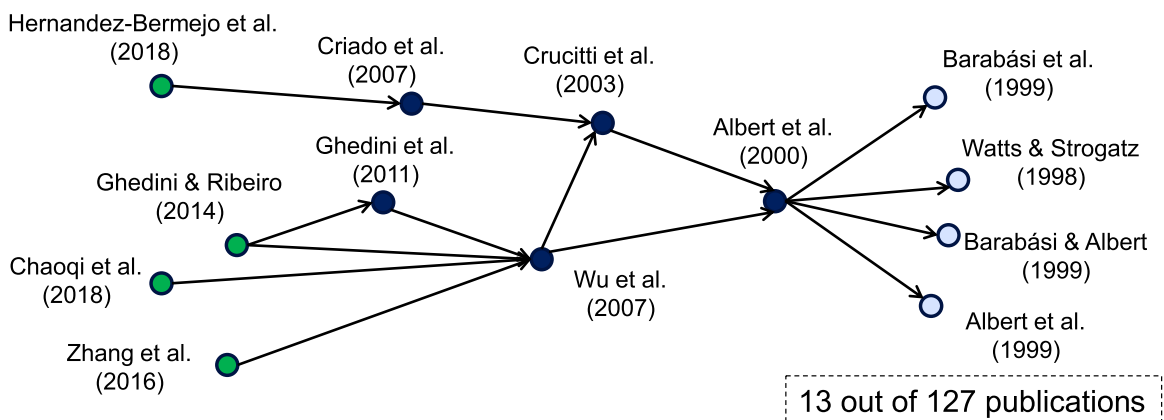

Fig. 11 Papers on topological vulnerability main paths 
documents and URL hyperlinks, respectively. They show that both of the in-degree and out-degree distributions follow a power-law. Barabási et al. (1999) investigate the properties of scale-free networks. They present a mean-field theory which can be used to predict dynamics of individual nodes and to calculate analytically the degree distribution. These sources are not directly related to vulnerability, but they provide widely applied concepts.

After these works, Albert et al. (2000) explore error and attack tolerance of networks by applying percolation theory. They investigate effects of removals of nodes/ edges on the network connectivity. They show that scale-free networks are highly robust against random failures. However, they are extremely vulnerable against targeted attacks on a few nodes with high degree. This paper by Albert et al. (2000) is the most-cited paper in the data set in this study.

There are mainly two streams after the paper by Albert et al. (2000). One stream is about network efficiency and vulnerability in scale-free networks. Crucitti et al. (2003) investigate the error and attack tolerance of scale-free networks with the concepts of global and local efficiency. They show that both of the global and local efficiency are not affected by random failures, but they are extremely vulnerable to attacks on highly connected nodes. Criado et al. (2007) analyze the correlation between efficiency, vulnerability and cost of networks and show strong correlations between them. Hernández-Bermejo et al. (2009) study the network efficiency of scale-free networks. They show that when a node is added to a network, the highest increase of network efficiency brought by the new node connects to the most connected existing node. They conclude that preferential attachment seems to lead to trade-off, more efficient network but more vulnerable against targeted attacks.

Another stream is about identifying critical nodes and defence strategies. Wu et al. (2007) introduce a model of intentional attacks with incomplete information. In this model, random failures and intentional attacks in Albert et al. (2000) are two extreme cases. They show that hiding a small fraction of nodes chosen randomly in scale-free networks can protect the whole network against intentional attacks. This is because a few hidden hubs can keep playing crucial roles for network connectivity. Ghedini and Ribeiro (2011) point out that vulnerability analysis based only on the centrality measurements may have some bias. They show that failures on lower centrality nodes sometimes have serious damage on the network connectivity. Ghedini and Ribeiro (2014) then propose strategies to identify vulnerable parts and to mitigate damage by changing network topology by rewiring or adding edges. The topics of the two sinks by Zhang et al. (2016c) and Chaoqi et al. (2018) are related to fuzzy evaluation of the vulnerability of networks and the vulnerability to attacks on multiple nodes, respectively.

In summary, papers in the main paths in this community mainly investigate the influences of the removals of nodes and/or edges randomly or intentionally. These studies analyze the static aspects of the vulnerability without considering network flow, which is different from papers in the cascading failures community as described in the next section. The pioneering work is the paper by Albert et al. (2000), which is the most-cited paper in this data set. The main finding is the robust, yet fragile property of scale-free networks. That is, scale-free networks are robust to random failures, but they are extremely fragile to intentional attacks on hubs. This 
property has been confirmed by using different methods and some defence strategies have been proposed. Methods proposed in these studies can be applied to any kind of network systems. In fact, some papers in the metro and maritime networks community apply these methods to transportation networks (especially to subway and railway networks). The three source papers, Watts and Strogatz (1998), Albert et al. (1999), and Albert et al. (2000), are published in Nature and the other source, Barabási and Albert (1999), is published in Science. Many of the other papers are published in Physica A: Statistical Mechanics and Its Applications.

\subsubsection{Cascading failures}

We show the main paths in the cascading failures community in Fig. 12. We identify 23 papers out of 110 publications.

In the topological vulnerability community, papers analyze the vulnerability of networks by iteratively removing nodes/edges without considering effects of network flow. However, in many network systems, the flow of some physical quantities is a crucial factor characterizing their vulnerability. In consideration of the existence of flow, the removals of nodes/edges redistribute the flow, possibly leading to catastrophic consequences by the domino effects. Typical examples are blackouts, communication disturbances, or financial crises. This kind of phenomena is called cascading failures.

There are six sources in the cascading failures main paths (Motter and Lai 2002; Wasserman and Faust 1994; Amaral et al. 2000; Albert et al. 2004; Kinney et al. 2005; Crucitti et al. 2005). Two papers by Wasserman and Faust (1994) and Amaral et al. (2000) are not directly related to cascading failures, but they present fundamental concepts in network theory. Wasserman and Faust (1994) cover various methodologies about social network analysis. This book includes concepts, such as centrality or cluster which are basic knowledge not only for social networks but also for

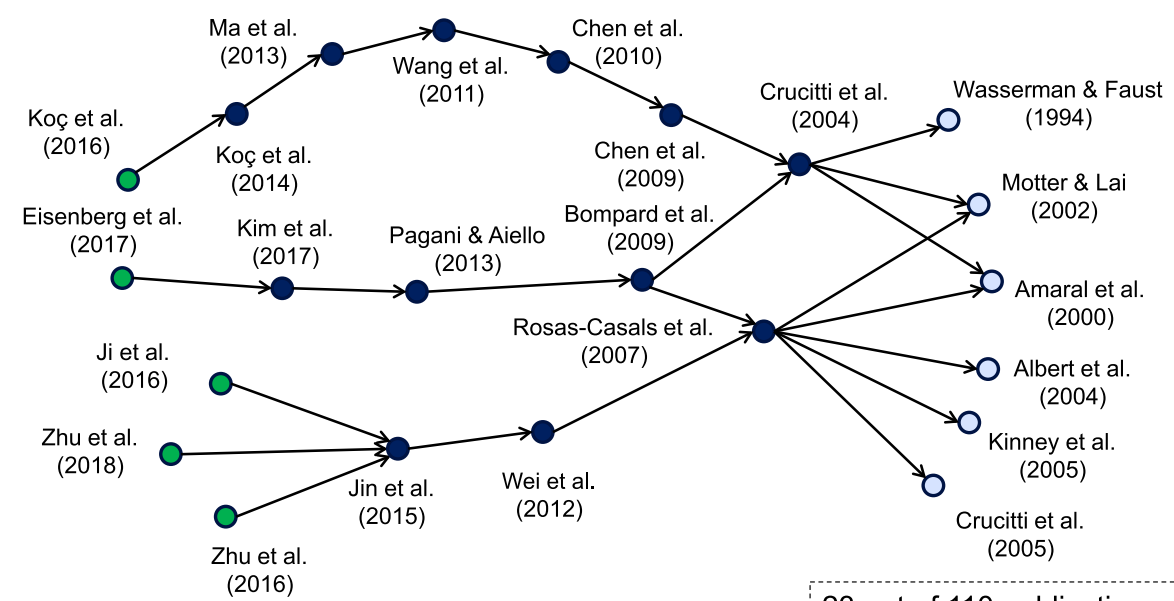

23 out of 110 publications

Fig. 12 Papers on cascading failures main paths 
any kind of networks. Amaral et al. (2000) analyze properties of diverse networks in the real world and show how different classes of small-world networks emerge. According to the obtained main paths, a pioneering work on cascading failures is a paper by Motter and Lai (2002). They propose a simple model for cascading failures where every node has a finite capacity to handle flow and overloaded nodes are successively removed from a network. They show that intentional attacks on a network with highly heterogeneous distribution of flow can easily lead to large-scale cascading failures. They also demonstrate that the breakdown of a single node with the largest load is sufficient to collapse the entire network. The other three papers by Albert et al. (2004), Kinney et al. (2005), and Crucitti et al. (2005), are related to blackout as cascading failures in power grids. Albert et al. (2004) study cascading failures in power grids in the background of large-scale blackout in the United States in 2003. They show that a power grid is robust against most failures, but breakdowns of some key nodes greatly reduce the function in the global scale. Kinney et al. (2005) analyze the robustness of the North American power grid against cascading failures. They show that the North American power grid is highly robust to random failures. However, targeted attacks on the few transmission substations with high betweenness and degree centrality cause serious damages on the entire system. Crucitti et al. (2005) further analyze the vulnerability of power grids in Spain, France, and Italy.

After these source papers, there are two papers in the middle of the 2000s (Crucitti et al. 2004; Rosas-Casals et al. 2007). Crucitti et al. (2004) propose an extended model based on the model proposed by Motter and Lai (2002) in the following two points: (1) overloaded nodes are not removed from a network, but flow avoids these nodes, and (2) damage is quantified by the decrease of network efficiency proposed by Latora and Marchiori (2001). They also demonstrate that the breakdown of a single node with the largest load is sufficient to collapse the entire network. RosasCasals et al. (2007) show that the robustness of 33 different European power grids which have exponential degree distributions is similar to the robustness of scale-free networks.

From about the year of 2010, there are three main streams in the main paths. The first stream starts with a paper by Chen et al. (2009). Papers on this stream are about the integration of methods between complex networks and electrical engineering. Chen et al. (2009) propose a model for structural vulnerability analysis of power grids based on Kirchoff's laws. Chen et al. (2010) further investigate the vulnerability of power grids. They improve traditional approaches by considering electrical power flow distribution instead of the shortest-path assignment. Their results indicate that a critical regime might exist where the network is vulnerable to both random failures and intentional attacks. Wang et al. (2011) define a new measure, electrical betweenness, by considering the maximum demand of load and the capacity of generators. They show that electrical betweenness distribution follows power-law and critical points can be identified as a node with high electrical betweenness. Ma et al. (2013) analyze the robustness of power grids with electrical efficiency. They show that the robustness does not always increase monotonically with capacity, which is different from some previous studies (e.g., Motter and Lai 2002; Crucitti et al. 2004; Koç et al. 2014). They propose a new metric, effective graph resistance, 
extended from the average shortest path length with the concept of the impedance. This metric can be used to estimate the robustness of a power grid against cascading failures. Koç et al. (2015) propose a measure to assess the robustness of power grids and apply it to a network constructed by real-world data.

The second stream begins with a paper by Bompard et al. (2009). Papers on this stream also analyze the power grids using the integrated methods between complex networks and electrical engineering. Bompard et al. (2009) propose extended metrics to overcome the disadvantages of purely topological metrics such as efficiency, degree or betweenness centrality for the vulnerability analysis of power grids. Pagani and Aiello (2013) present a survey of studies about properties of different power grids in the United States, Europe, and China. Highlights of this survey are as follows: (1) degree distributions in power grids tend to follow an exponential distribution, (2) very few nodes have high betweenness centrality, which is similar to properties of networks with power-law degree distributions, (3) power grids are robust to random failures, but extremely vulnerable to targeted attacks on critical parts such as nodes with high degree or nodes and edges with high betweenness centrality, and (4) results obtained by complex network analysis are very close to the ones obtained by traditional electrical engineering. Kim and Ryerson (2017) analyze the South Korean power grid. Their results show that the South Korean power grid is more vulnerable compared to ER random networks (Erdos and Rényi 1960) and BA scale-free networks (Barabási and Albert 1999). Eisenberg et al. (2017) develop a method to improve response for blackouts and identified critical components in power grids.

The third stream starts from a paper by Wei et al. (2012). This stream is slightly different from the other two streams. Many papers in the other two streams aim to integrate the methods in complex networks and electrical engineering. On the other hand, papers in the third stream tend to develop models for cascading failures in more general networks. They propose a model for cascading failures with the local preferential redistribution rule. The load of a broken node is redistributed to neighbour nodes depending on their degree. Jin et al. (2015) propose a model for cascading failures in directed and weighted networks. Zhu et al. (2016) extend a model for cascading failures with fuzzy information to consider with uncertain failures or attacks. Zhu et al. (2018) investigate the way to improve robustness against cascading failures with two multi-objective optimization problems which consider operational cost and robustness. Ji et al. (2016) analyze the structure of the global electricity trade network with nations as nodes and international electricity trade as edges.

Summing up the above, papers in the cascading failures main paths investigate the vulnerability of network systems in consideration with the network flow. Removals of nodes/edges redistribute the network flow and possibly cause the propagation of failures in the entire network. After the pioneering work by Motter and Lai (2002), models for cascading failures have been developed in different ways. Many papers show that the breakdown of only a few nodes with high centrality measures cause devastating cascading failures in the whole network. This is a consistent result as findings in the papers in the topological vulnerability community show. In addition, many papers in the obtained main paths analyze blackouts as cascading failures in power grids and some papers discuss the integration of concepts and methods 


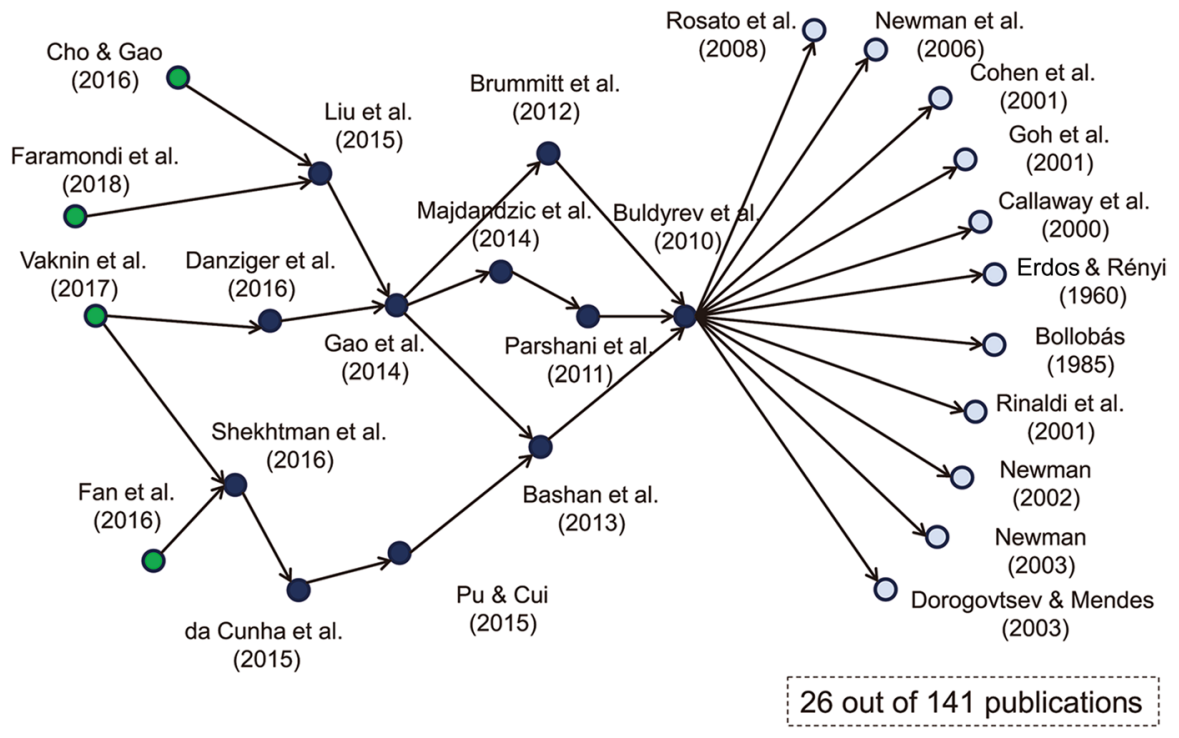

Fig. 13 Papers on interdependent networks main paths

between complex networks and electrical engineering. An interesting finding is that the results obtained from traditional electrical engineering and complex networks are similar. Many papers in the main paths are published in complex network-related journals such as Physical Review E and Physica A: Statistical Mechanics and Its Applications.

\subsubsection{Interdependent networks}

We show the main paths in the interdependent networks community in Fig. 13. We identify 26 papers out of 141 publications.

There are 11 source papers in the obtained main paths (Rinaldi et al. 2001; Erdos and Rényi 1960; Callaway et al. 2000; Bollobás and Béla 2001; Cohen et al. 2001; Goh et al. 2001; Newman 2002, 2003; Dorogovtsev and Mendes 2013; Newman et al. 2006; Rosato et al. 2008). All of these papers are cited by a pioneering paper on the vulnerability of interdependent networks by Buldyrev et al. (2010). There are three books and one review paper which summarize the fundamental theory of complex networks (Bollobás and Béla 2001; Newman 2003; Dorogovtsev and Mendes 2013; Newman et al. 2006). The contents of the other sources are various such as random graphs (Erdos and Rényi 1960), percolation theory (Callaway et al. 2000; Cohen et al. 2001), betweenness distribution (Goh et al. 2001), disease spreading (Newman 2002), interdependency between critical infrastructures (Rinaldi et al. 2001; Rosato et al. 2008).

Before the paper by Buldyrev et al. (2010) in 2010, most studies in complex networks analyzed the vulnerability of a single network. However, as Rinaldi et al. (2001) point out, most critical infrastructures have interdependency among them. 
This indicates that influences of failures in one system can propagate through dependency and other systems can be also seriously damaged. To study the influences of such an interdependency on the vulnerability of networks, Buldyrev et al. (2010) propose a model for cascading failures in interdependent networks. They show that interdependency makes the system vulnerable and they behave totally differently when they are isolated networks. They conclude that it is necessary to consider interdependency to assess the vulnerability of network systems.

After the paper by Buldyrev et al. (2010), methods to analyze the vulnerability of interdependent networks have been extended in different ways. There are four papers (Brummitt et al. 2012; Parshani et al. 2011; Bashan et al. 2013; Majdandzic et al. 2014) toward a paper by Gao et al. (2014). Brummitt et al. (2012) show that there is an optimal fraction of interconnected node pairs between two mutually dependent networks which minimizes the size of cascading failures. Parshani et al. (2011) introduce two types of edges, connectivity edges and dependency edges, and propose an analytical framework to evaluate the vulnerability of interdependent networks. Connectivity edges are the ones in each network and dependency edges are the ones between different networks. They show that high density of the dependency edges makes the network vulnerable. Because many infrastructure network systems are spatially embedded, Bashan et al. (2013) study the vulnerability of spatially embedded interdependent networks. They show that spatially embedded networks are more vulnerable compared to non-embedded networks. Majdandzic et al. (2014) analyze spontaneous recovery of network systems after disrupted events. Even though this study is not directly related to interdependent networks, Gao et al. (2014) mention that the recovery of damaged interdependent networks is a possible future research direction.

All of the above-mentioned papers are cited by the review paper by Gao et al. (2014). They review studies on the vulnerability of interdependent networks. They show that many data sets can be represented as network of networks including networks of different transportation networks such as flight networks, railway networks and road networks. They summarize some interesting and surprising findings on the vulnerability of interdependent networks and they conclude that one possible research direction is to develop methods to improve the robustness of spatially embedded interdependent networks. After the paper by Gao et al. (2014), there is one review paper (Liu et al. 2015) and one book chapter (Danziger et al. 2016). Liu et al. (2015) review the framework for analyzing interdependent networks. They show that there are two strategies to decrease the vulnerability of interdependent networks: (1) protecting high-degree nodes, and (2) increasing the degree correlation between networks. A book chapter by Danziger et al. (2016) also reviews the vulnerability of interdependent networks including discussions about the role of connectivity and dependency edges in mutually dependent networks. The topics of the sink papers are related to temporal networks (Cho and Gao 2016) and the vulnerability of interdependent networks based on the concept of controllability (Faramondi et al. 2018).

From the paper by Bashan et al. (2013), we observe one stream. Pu and Cui (2015) study the longest-path attack on networks and they show that homogeneous networks are vulnerable to the longest-path attacks. Da Cunha et al. (2015) propose 
a module-based method to attack networks. This method outperforms other ways of attacks based on degree or betweenness centrality. Shekhtman et al. (2016) review studies on failure and recovery in interdependent networks. They point out that, even though it is found that spontaneous recovery can occur in a single network (Majdandzic et al. 2014), recovery in interdependent networks is more complicated and it should be further studied. The topics of the two sink papers are related to the vulnerability of spatially embedded two-interdependent networks (Vaknin et al. 2017) and the vulnerability of two-interdependent networks which are operated by two different entities (Fan et al. 2017).

Summing up the above, after the paper by Buldyrev et al. (2010), studies on interdependent networks have been developed rapidly in the 2010s. Many studies have reported that interdependent networks behave completely different from single networks and they are more vulnerable compared to such isolated networks. It is more difficult to protect interdependent networks compared to single networks. Moreover, in recent years, some papers show that spatially embedded interdependent networks are much more vulnerable than non-embedded ones. Transportation networks are often taken as examples of such spatial embedded interdependent networks. Some papers point out that many studies should pursue defence or recovery strategies for spatially embedded interdependent networks (e.g., Bashan et al. 2013; Gao et al. 2014; Vaknin et al. 2017). We expect that interdisciplinary studies between complex networks and transportation engineering should contribute to construct strategies to protect spatially embedded interdependent networks in the future. In the main paths in this community, many papers are published in complex network-related journals such as Physical Review Letters or Physical Review E.

\subsection{Citation patterns among different communities}

Finally, we discuss the relationships among the identified communities by visualizing the Sankey diagram. In Fig. 14, we show the Sankey diagram of intra/intercommunity edges among seven communities. The numbers shown in the left side represent the number of the citing articles and the ones shown in the right side represent the number of the cited articles. Hence, the total value of each side is equal to the number of edges in the GWCC, 4584.

The first thing to note about Fig. 14 is that we observe asymmetric citation patterns between the two fields of transportation and complex networks, when we assume that the three communities (transport vulnerability, metro and maritime networks, and resilience) are transportation-related communities and the others (topological vulnerability, cascading failures, and interdependent networks) are complex network-related communities. Especially, papers in the topological vulnerability are cited well by all the other communities. This result is intuitive, because not only the most cited papers (e.g., Watts and Strogatz 1998; Barabási and Albert 1999; Albert et al. 2000) are included in this community but also some papers in the topological vulnerability propose the methods to evaluate the topological vulnerability of any kind of networks. These methods can be directly applied to transportation networks. Actually, papers in the metro and maritime networks apply these methods 


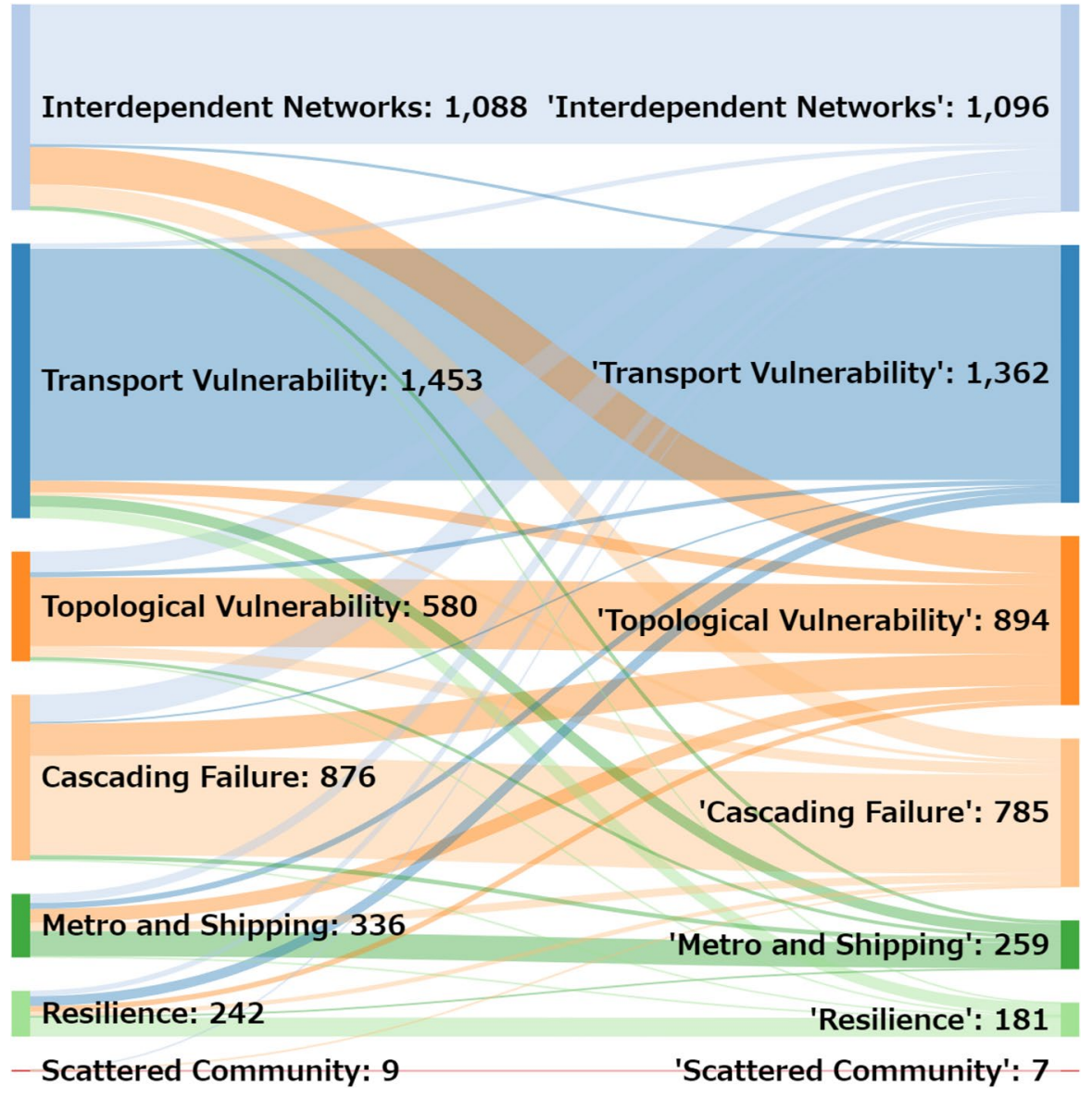

Fig. 14 Citation patterns among identified communities. The numbers of left and right sides represent the number of the citing and cited articles, respectively

to evaluate the topological vulnerability of subway, railway, or maritime shipping networks. In addition, as we observe in the main paths in the transport vulnerability, the vulnerability of public transportation networks (PTNs) has rapidly grown after the successful integration of the concepts between complex networks and transportation engineering (Cats and Jenelius 2014). Moreover, the papers in the resilience community analyze the resilience of networks with hubs based on the results in complex networks (e.g., Reggiani 2013; O’Kelly 2015).

The second thing to note about Fig. 14 is that there are few citations between the transport vulnerability and cascading failures communities in both directions. Papers in the cascading failures study how failures propagate in network systems. However, most papers focus on only blackouts in power grids. That is, universal and specific properties of cascading failures across different network systems have not been discussed deeply. In the transport vulnerability community, only a few recent 
papers try to integrate the methods in complex networks with specific natures of the PTNs to understand how these systems are disrupted (e.g., Cats and Jenelius 2014; Cats 2016; Malandri et al. 2018). More studies should be required in this direction and it also should be discussed in association with cascading failures in other network systems.

The third point to note about Fig. 14 is that there are few citations between transportation-related communities and the interdependent networks community. As described in the main paths in the interdependent networks community, the vulnerability of interdependent networks has been studied intensively in the last decade. Some recent papers show that spatially embedded interdependent networks are extremely vulnerable with transportation networks as typical examples and it is also pointed out that defence strategies for such networks should be studied more deeply (e.g., Bashan et al. 2013; Gao et al. 2014; Vaknin et al. 2017). However, these studies do not consider specific natures of transportation networks in both of the demand and supply sides. More studies are desirable to understand influences of these specific properties on the vulnerability of spatially embedded interdependent networks.

\section{Conclusions}

In this study, we have comprehensively and objectively revealed the overall perspectives of the vulnerability studies in the fields of transportation and complex networks by applying the citation network analysis. We collected publication records from the online publication database, the Web of Science, and we constructed the citation network. Then we analyzed the giant weakly connected component consisting of 705 nodes (publications) and 4,584 directed edges (citation relations). The proposed framework can be applicable to any research fields. Our main results and implications are summarized as follows:

1. We have identified seven major research domains: (1) transport vulnerability, (2) metro and maritime networks, (3) resilience, (4) topological vulnerability, (5) cascading failures, (6) interdependent networks, and (7) scattered community.

2. We have identified the major research backbones in each of the detected research domains and briefly described how research has been developed over time.

3. We have shown that, among various transportation modes, studies on the vulnerability of maritime networks are still limited and more studies are desirable in the future.

4. We have quantitatively revealed the asymmetrical citation patterns between the fields of transportation and complex networks, which implies that the mutual understanding between them is currently lacking.

5. We have shown that there are few citations between the transport vulnerability and cascading failures communities in both directions. This result suggests that the findings in the two communities have not been discussed deeply, even though they study the dynamical aspects of the vulnerability of network systems in general. 
6. We have shown that there are few citations between transportation-related communities and the interdependent networks community. The vulnerability of spatially embedded interdependent networks has been studied in complex networks in recent years with transportation networks as typical examples. However, these studies usually do not consider influences of specific natures of transportation networks in both of the supply and demand sides. More studies are desirable to understand influences of these specific properties on the vulnerability of spatially embedded interdependent networks.

Acknowledgements This work was supported by JSPS KAKENHI Grant-in-Aid for Research Activity start-up \#19K23531, and JSPS KAKENHI Grant-in-Aid for Scientific Research (A) \#17H01297.

\section{Compliance with ethical standards}

Conflict of interest The authors declare that they have no competing interests.

Open Access This article is licensed under a Creative Commons Attribution 4.0 International License, which permits use, sharing, adaptation, distribution and reproduction in any medium or format, as long as you give appropriate credit to the original author(s) and the source, provide a link to the Creative Commons licence, and indicate if changes were made. The images or other third party material in this article are included in the article's Creative Commons licence, unless indicated otherwise in a credit line to the material. If material is not included in the article's Creative Commons licence and your intended use is not permitted by statutory regulation or exceeds the permitted use, you will need to obtain permission directly from the copyright holder. To view a copy of this licence, visit http://creativecommons.org/licen ses/by/4.0/.

\section{References}

Albert R, Jeong H, Barabási A-L (1999) Internet: diameter of the world-wide web. Nature 401(6749):130

Albert R, Jeong H, Barabási A-L (2000) Error and attack tolerance of complex networks. Nature 406(6794):378

Albert R, Albert I, Nakarado GL (2004) Structural vulnerability of the North American power grid. Phys Rev E 69(2):025103

Amaral LAN, Scala A, Barthelemy M, Stanley HE (2000) Classes of small-world networks. Proc Natl Acad Sci 97(21):11149-11152

Angeloudis P, Fisk D (2006) Large subway systems as complex networks. Phys A Stat Mech Appl 367:553-558

Asakura Y (1999) Evaluation of network reliability using stochastic user equilibrium. J Adv Transp 33(2):147-158

Barabási A-L (2016) Network science. Cambridge University Press, Cambridge

Barabási A-L, Albert R (1999) Emergence of scaling in random networks. Science 286(5439):509-512

Barabási A-L, Albert R, Jeong H (1999) Mean-field theory for scale-free random networks. Phys A Stat Mech Appl 272(1-2):173-187

Bashan A, Berezin Y, Buldyrev SV, Havlin S (2013) The extreme vulnerability of interdependent spatially embedded networks. Nat Phys 9(10):667

Batagelj V (2003) Efficient algorithms for citation network analysis. arXiv preprint: cs/0309023

Bell MG (2000) A game theory approach to measuring the performance reliability of transport networks. Transp Res Part B Methodol 34(6):533-545

Bell MG, Iida Y (1997) Transportation network analysis. Wiley, Chichester 
Bell MG, Kurauchi F, Perera S, Wong W (2017) Investigating transport network vulnerability by capacity weighted spectral analysis. Transp Res Part B Methodol 99:251-266

Blondel VD, Guillaume J-L, Lambiotte R, Lefebvre E (2008) Fast unfolding of communities in large networks. J Stat Mech Theory Exp 2008(10):P10008

Bollobás B, Béla B (2001) Random Graphs. Number 73. Cambridge University Press, Cambridge

Bompard E, Napoli R, Xue F (2009) Analysis of structural vulnerabilities in power transmission grids. Int J Crit Infrastruct Protect 2(1-2):5-12

Brown G, Carlyle M, Salmerón J, Wood K (2006) Defending critical infrastructure. Interfaces 36(6):530-544

Brummitt CD, D'Souza RM, Leicht EA (2012) Suppressing cascades of load in interdependent networks. Proc Natl Acad Sci 109(12):E680-E689

Bruneau M, Chang SE, Eguchi RT, Lee GC, O’Rourke TD, Reinhorn AM, Shinozuka M, Tierney K, Wallace WA, Von Winterfeldt D (2003) A framework to quantitatively assess and enhance the seismic resilience of communities. Earthquake Spectra 19(4):733-752

Buldyrev SV, Parshani R, Paul G, Stanley HE, Havlin S (2010) Catastrophic cascade of failures in interdependent networks. Nature 464(7291):1025-1028

Callaway DS, Newman ME, Strogatz SH, Watts DJ (2000) Network robustness and fragility: percolation on random graphs. Phys Rev Lett 85(25):5468

Cats O (2016) The robustness value of public transport development plans. J Transport Geogr 51:236-246

Cats O, Jenelius E (2014) Dynamic vulnerability analysis of public transport networks: mitigation effects of real-time information. Netw Spatial Econ 14(3-4):435-463

Cats O, Jenelius E (2015) Planning for the unexpected: the value of reserve capacity for public transport network robustness. Transp Res Part A Policy Pract 81:47-61

Chaoqi F, Ying W, Xiaoyang W, Yangjun G (2018) Multi-node attack strategy of complex networks due to cascading breakdown. Chaos Solit Fractals 106:61-66

Chen A, Yang H, Lo HK, Tang WH (1999) A capacity related reliability for transportation networks. J Adv Transport 33(2):183-200

Chen A, Yang H, Lo HK, Tang WH (2002) Capacity reliability of a road network: an assessment methodology and numerical results. Transport Res Part B Methodol 36(3):225-252

Chen A, Yang C, Kongsomsaksakul S, Lee M (2007) Network-based accessibility measures for vulnerability analysis of degradable transportation networks. Netw Spatial Econ 7(3):241-256

Chen G, Dong ZY, Hill DJ, Zhang GH (2009) An improved model for structural vulnerability analysis of power networks. Phys A Stat Mech Appl 388(19):4259-4266

Chen G, Dong ZY, Hill DJ, Zhang GH, Hua KQ (2010) Attack structural vulnerability of power grids: a hybrid approach based on complex networks. Phys A Stat Mech Appl 389(3):595-603

Cho J-H, Gao J (2016) Cyber war game in temporal networks. PLOS One 11(2):e0148674

Cohen R, Erez K, Ben-Avraham D, Havlin S (2001) Breakdown of the internet under intentional attack. Phys Rev Lett 86(16):3682

Cox A, Prager F, Rose A (2011) Transportation security and the role of resilience: a foundation for operational metrics. Transport Policy 18(2):307-317

Criado R, Hernandez-Bermejo B, Romance M (2007) Efficiency, vulnerability and cost: an overview with applications to subway networks worldwide. Int J Bifurcation Chaos 17(07):2289-2301

Crucitti P, Latora V, Marchiori M, Rapisarda A (2003) Efficiency of scale-free networks: error and attack tolerance. Phys A Stat Mech Appl 320:622-642

Crucitti P, Latora V, Marchiori M (2004) Model for cascading failures in complex networks. Phys Rev E 69(4):045104

Crucitti P, Latora V, Marchiori M (2005) Locating critical lines in high-voltage electrical power grids. Fluctuation Noise Lett 5(02):L201-L208

da Cunha BR, González-Avella JC, Gonçalves S (2015) Fast fragmentation of networks using modulebased attacks. PLOS One 10(11):e0142824

Danziger MM, Shekhtman LM, Bashan A, Berezin Y, Havlin S (2016) Vulnerability of interdependent networks and networks of networks. In: Interconnected networks, pp 79-99. Springer, New York

Derrible S, Kennedy C (2010) The complexity and robustness of metro networks. Phys A Stat Mech Appl 389(17):3678-3691

D'Este G, Taylor MA (2003) Network vulnerability: an approach to reliability analysis at the level of national strategic transport networks. In: The network reliability of transport: Proceedings of the 1st international symposium on transportation network reliability (INSTR), pp 23-44. Emerald Group Publishing Limited 
Dijkstra EW (1959) A note on two problems in connexion with graphs. Numer Math 1(1):269-271

Dorogovtsev SN, Mendes JF (2013) Evolution of networks: from biological nets to the internet and WWW. OUP, Oxford

Ducruet C (2016) The polarization of global container flows by interoceanic canals: geographic coverage and network vulnerability. Maritime Policy Manag 43(2):242-260

D'Este GM, Taylor MA (2001) Modelling network vulnerability at the level of the national strategic transport network. J Eastern Asia Soc Transport Stud 4(2):1-14

Eisenberg DA, Park J, Seager TP (2017) Sociotechnical network analysis for power grid resilience in South Korea. Complexity 2017:1-14

Erdos P, Rényi A (1960) On the evolution of random graphs. Publ Math Inst Hung Acad Sci 5(1):17-60

Fan Y, Cao G, He S, Chen J, Sun Y (2017) Game among interdependent networks: the impact of rationality on system robustness. EPL (Europhys Lett) 116(6):68002

Faramondi L, Oliva G, Piemonte V (2018) Novel vulnerability metrics for interdependent system based on system controllability. J Phys Conf Ser 1026:012021 (IOP Publishing)

Fortunato S, Barthelemy M (2007) Resolution limit in community detection. Proc Natl Acad Sci 104(1):36-41

Franchin P, Cavalieri F (2015) Probabilistic assessment of civil infrastructure resilience to earthquakes. Comput-Aided Civil Infrastruct Eng 30(7):583-600

Fu G, Wilkinson S, Dawson RJ (2016) A spatial network model for civil infrastructure system development. Comput-Aided Civil Infrastruct Eng 31(9):661-680

Gao J, Li D, Havlin S (2014) From a single network to a network of networks. Natl Sci Rev 1(3):346-356

Ghedini CG, Ribeiro CH (2011) Rethinking failure and attack tolerance assessment in complex networks. Phys A Stat Mech Appl 390(23-24):4684-4691

Ghedini CG, Ribeiro CH (2014) Improving resilience of complex networks facing attacks and failures through adaptive mechanisms. Adv Complex Syst 17(02):1450009

Goh K-I, Kahng B, Kim D (2001) Universal behavior of load distribution in scale-free networks. Phys Rev Lett 87(27):278701. https://doi.org/10.1103/PhysRevLett.87.278701

Griffith DA, Chun Y (2015) Spatial autocorrelation in spatial interactions models: geographic scale and resolution implications for network resilience and vulnerability. Netw Spatial Econ 15(2):337-365

Heilig L, Voß S (2015) A scientometric analysis of public transport research. J Public Transport 18(2):111-141. https://doi.org/10.5038/2375-0901.18.2.8

Helbing D (2013) Globally networked risks and how to respond. Nature 497(7447):51-59. https://doi. org/10.1038/nature12047

Hernández-Bermejo B, Marco-Blanco J, Romance M (2009) Analytical estimates and proof of the scale-free character of efficiency and improvement in Barabási-Albert trees. Phys Lett A 373(8-9):838-843

Holling CS (1973) Resilience and stability of ecological systems. Annu Rev Ecol Syst 4(1):1-23

Hummon NP, Dereian P (1989) Connectivity in a citation network: the development of dna theory. Soc Netw 11(1):39-63

Jenelius E (2009) Network structure and travel patterns: explaining the geographical disparities of road network vulnerability. J Transport Geogr 17(3):234-244

Jenelius E (2010) Redundancy importance: links as rerouting alternatives during road network disruptions. Proc Eng 3:129-137

Jenelius E, Cats O (2015) The value of new public transport links for network robustness and redundancy. Transportmetrica A: Transport Sci 11(9):819-835. https://doi.org/10.1080/23249935.2015.10872 32

Jenelius E, Mattsson L-G (2012) Road network vulnerability analysis of area-covering disruptions: a grid-based approach with case study. Transport Res Part A Policy Pract 46(5):746-760

Jenelius E, Petersen T, Mattsson L-G (2006) Importance and exposure in road network vulnerability analysis. Transport Res Part A Policy Pract 40(7):537-560

Ji L, Jia X, Chiu AS, Xu M (2016) Global electricity trade network: structures and implications. PLOS One 11(8):e0160869

Jiang C, Bhat CR, Lam WH (2020) A bibliometric overview of transportation research part B: methodological in the past forty years (1979-2019). Transport Res Part B Methodol 138:268-291

Jin L, Wang H, Xie B, Yu L, Liu L (2017) A user exposure based approach for non-structural road network vulnerability analysis. PLOS One 12(11):e0188790

Jin W-X, Song P, Liu G-Z, Stanley HE (2015) The cascading vulnerability of the directed and weighted network. Phys A: Stat Mech Appl 427:302-325 
Johansson J, Hassel H (2010) An approach for modelling interdependent infrastructures in the context of vulnerability analysis. Reliab Eng Syst Saf 95(12):1335-1344

Karakose G, McGarvey RG (2018) Capacitated path-aggregation constraint model for arc disruption in networks. Transp Res Part E Logist Transp Rev 109:225-238

Kim DH, Eisenberg DA, Chun YH, Park J (2017) Network topology and resilience analysis of South Korean power grid. Phys A: Stat Mech Appl 465:13-24

Kim H, Ryerson MS (2017) The q-ad hoc hub location problem for multi-modal networks. Netw Spatial Econ 17(3):1015-1041

Kinney R, Crucitti P, Albert R, Latora V (2005) Modeling cascading failures in the north american power grid. Eur Phys J B Condens Matter Complex Syst 46(1):101-107

Koç Y, Warnier M, Van Mieghem P, Kooij RE, Brazier FM (2014) The impact of the topology on cascading failures in a power grid model. Phys A: Stat Mech Appl 402:169-179

Koç Y, Raman A, Warnier M, Kumar T (2015) Structural vulnerability analysis of electric power distribution grids. arXiv preprint arXiv:1506.08641

Latora V, Marchiori M (2001) Efficient behavior of small-world networks. Phys Rev Lett 87(19):198701

Latora V, Marchiori M (2002) Is the Boston subway a small-world network? Phys A: Stat Mech Appl 314(1-4):109-113. https://doi.org/10.1016/S0378-4371(02)01089-0

Liu JS, Lu LY (2012) An integrated approach for main path analysis: development of the Hirsch index as an example. J Am Soc Inf Sci Technol 63(3):528-542

Liu X, Peng H, Gao J (2015) Vulnerability and controllability of networks of networks. Chaos Solit Fractals 80:125-138

Lu Q-C, Zhang J, Peng Z-R, Rahman AS (2014) Inter-city travel behaviour adaptation to extreme weather events. J Transport Geogr 41:148-153

Ma T-L, Yao J-X, Qi C, Zhu H-L, Sun Y-S (2013) Non-monotonic increase of robustness with capacity tolerance in power grids. Phys A Stat Mech Appl 392(21):5516-5524

Mahmoud H, Chulahwat A (2018) Spatial and temporal quantification of community resilience: Gotham city under attack. Comput-Aided Civil Infrastruct Eng 33(5):353-372

Majdandzic A, Podobnik B, Buldyrev SV, Kenett DY, Havlin S, Stanley HE (2014) Spontaneous recovery in dynamical networks. Nat Phys 10(1):34

Malandri C, Fonzone A, Cats O (2018) Recovery time and propagation effects of passenger transport disruptions. Phys A: Stat Mech Appl 505:7-17

Mattsson L-G, Jenelius E (2015) Vulnerability and resilience of transport systems-a discussion of recent research. Transp Res Part A Policy Pract 81:16-34

Modak NM, Merigó JM, Weber R, Manzor F, de Dios Ortúzar J (2019) Fifty years of transportation research journals: a bibliometric overview. Transport Res Part A Policy Pract 120:188-223

Motter AE, Lai Y-C (2002) Cascade-based attacks on complex networks. Phys Rev E 66(6):065102

Murray AT, Matisziw TC, Grubesic TH (2008) A methodological overview of network vulnerability analysis. Growth Change 39(4):573-592

Newman M, Barabasi A-L, Watts DJ (2006) The structure and dynamics of networks (Princeton studies in complexity)

Newman ME (2002) Spread of epidemic disease on networks. Phys Rev E 66(1):016128

Newman ME (2003) The structure and function of complex networks. SIAM Rev 45(2):167-256

Nicholson A, Schmöcker J-D, Bell MG, Iida Y (2003) Assessing transport reliability: malevolence and user knowledge. In: The network reliability of transport: Proceedings of the 1st international symposium on transportation network reliability (INSTR), pp 1-22. Emerald Group Publishing Limited

Östh J, Dolciotti M, Reggiani A, Nijkamp P (2018) Social capital, resilience and accessibility in urban systems: a study on Sweden. Netw Spatial Econ 18(2):313-336

Ouyang M, Fang Y (2017) A mathematical framework to optimize critical infrastructure resilience against intentional attacks. Comput-Aided Civil Infrastruct Eng 32(11):909-929

Ouyang M, Tao F, Huang S, Xu M, Zhang C (2018) Vulnerability mitigation of multiple spatially localized attacks on critical infrastructure systems. Comput-Aided Civil Infrastruct Eng 33(7):585-601

O’Kelly ME (2015) Network hub structure and resilience. Netw Spatial Econ 15(2):235-251

Pagani GA, Aiello M (2013) The power grid as a complex network: a survey. Phys A: Stat Mech Appl 392(11):2688-2700

Parshani R, Buldyrev SV, Havlin S (2011) Critical effect of dependency groups on the function of networks. Proc Natl Acad Sci 108(3):1007-1010 
Pimm SL (1984) The complexity and stability of ecosystems. Nature 307(5949):321

Pu C-L, Cui W (2015) Vulnerability of complex networks under path-based attacks. Phys A: Stat Mech Appl 419:622-629

Reggiani A (2013) Network resilience for transport security: Some methodological considerations. Transport Policy 28:63-68

Reggiani A, Nijkamp P, Lanzi D (2015) Transport resilience and vulnerability: the role of connectivity. Transport Res Part A Policy Pract 81:4-15

Rinaldi SM, Peerenboom JP, Kelly TK (2001) Identifying, understanding, and analyzing critical infrastructure interdependencies. IEEE Control Syst 21(6):11-25

Rodríguez-Núñez E, García-Palomares JC (2014) Measuring the vulnerability of public transport networks. J Transport Geogr 35:50-63

Rosas-Casals M, Valverde S, Solé RV (2007) Topological vulnerability of the European power grid under errors and attacks. Int J Bifurcation Chaos 17(07):2465-2475

Rosato V, Issacharoff L, Tiriticco F, Meloni S, Porcellinis S, Setola R (2008) Modelling interdependent infrastructures using interacting dynamical models. Int J Crit Infrastruct 4(1-2):63-79

Schmidt M (2008) The Sankey diagram in energy and material flow management: part ii: methodology and current applications. J Ind Ecol 12(2):173-185

Shekhtman LM, Danziger MM, Havlin S (2016) Recent advances on failure and recovery in networks of networks. Chaos Solit Fractals 90:28-36

Shelat S, Cats O (2017) Measuring spill-over effects of disruptions in public transport networks. In: 2017 5th IEEE international conference on models and technologies for intelligent transportation systems (MT-ITS), pp 756-761. IEEE, New York

Sullivan J, Aultman-Hall L, Novak D (2009) A review of current practice in network disruption analysis and an assessment of the ability to account for isolating links in transportation networks. Transport Lett 1(4):271-280

Sullivan J, Novak D, Aultman-Hall L, Scott DM (2010) Identifying critical road segments and measuring system-wide robustness in transportation networks with isolating links: a link-based capacity-reduction approach. Transport Res Part A Policy Pract 44(5):323-336

Taylor M (2017) Vulnerability analysis for transportation networks. Elsevier, London

Taylor MA (2008) Critical transport infrastructure in urban areas: impacts of traffic incidents assessed using accessibility-based network vulnerability analysis. Growth Change 39(4):593-616

Taylor MA, D'Este GM (2007) Transport network vulnerability: a method for diagnosis of critical locations in transport infrastructure systems. In: Critical infrastructure, pp 9-30. Springer, New York

Taylor MA, Sekhar SV, D'Este GM (2006) Application of accessibility based methods for vulnerability analysis of strategic road networks. Netw Spatial Econ 6(3-4):267-291

Vaknin D, Danziger MM, Havlin S (2017) Spreading of localized attacks in spatial multiplex networks. New J Phys 19(7):073037

Van Eck NJ, Waltman L (2014) Citnetexplorer: a new software tool for analyzing and visualizing citation networks. J Inform 8(4):802-823

Viljoen NM, Joubert JW (2016) The vulnerability of the global container shipping network to targeted link disruption. Phys A Stat Mech Appl 462:396-409

Viljoen NM, Joubert JW (2018) The road most travelled: the impact of urban road infrastructure on supply chain network vulnerability. Netw Spatial Econ 18(1):85-113

Wang K, Zhang B-H, Zhang Z, Yin X-G, Wang B (2011) An electrical betweenness approach for vulnerability assessment of power grids considering the capacity of generators and load. Phys A Stat Mech Appl 390(23-24):4692-4701

Wasserman S, Faust K (1994) Social network analysis: methods and applications, Vol 8. Cambridge University Press, Cambridge

Watts DJ, Strogatz SH (1998) Collective dynamics of 'small-world'networks. Nature 393(6684):440

Wei DQ, Luo XS, Zhang B (2012) Analysis of cascading failure in complex power networks under the load local preferential redistribution rule. Phys A Stat Mech Appl 391(8):2771-2777

Wu J, Deng H-Z, Tan Y-J, Zhu D-Z (2007) Vulnerability of complex networks under intentional attack with incomplete information. J Phys A Math Theor 40(11):2665

Xu X, Chen A, Yang C (2017) An optimization approach for deriving upper and lower bounds of transportation network vulnerability under simultaneous disruptions of multiple links. Transport Res Proc 23:645-663 
Xu W, Zhou J, Qiu G (2018) China's high-speed rail network construction and planning over time: a network analysis. J Transport Geogr 70:40-54

Zavitsas K, Bell MG (2010) A simulation model and a vulnerability assessment of the worldwide energy supply. In: Security and environmental sustainability of multimodal transport, pp 227246. Springer, New York

Zhang J, Xu X, Hong L, Wang S, Fei Q (2011) Networked analysis of the Shanghai subway network, in China. Phys A: Stat Mech Appl 390(23-24):4562-4570

Zhang J, Wang S, Zhang Z, Zou K, Shu Z (2016a) Characteristics on hub networks of urban rail transit networks. Phys A: Stat Mech Appl 447:502-507

Zhang J, Hu F, Wang S, Dai Y, Wang Y (2016b) Structural vulnerability and intervention of high speed railway networks. Phys A: Stat Mech Appl 462:743-751

Zhang J, Wang S, Wang X (2018) Comparison analysis on vulnerability of metro networks based on complex network. Phys A: Stat Mech Appl 496:72-78

Zhang R, Ran X, Wang C, Deng Y (2016c) Fuzzy evaluation of network vulnerability. Qual Reliab Eng Int 32(5):1715-1730

Zhang X-T, Bi H-L, Wang Y (2017) A stochastic programming approach for resilient hub location in power projection network considering random hub failures. Math Probl Eng 2017:6517453. https://doi.org/10.1155/2017/6517453

Zhu Q, Zhu Z, Wang Y, Yu H (2016) Fuzzy-information-based robustness of interconnected networks against attacks and failures. Phys A: Stat Mech Appl 458:194-203

Zhu Q, Zhu Z, Qi Y, Yu H, Xu Y (2018) Optimization of cascading failure on complex network based on NNIA. Phys A: Stat Mech Appl 501:42-51

Publisher's Note Springer Nature remains neutral with regard to jurisdictional claims in published maps and institutional affiliations. 